\title{
Analytical integrations in 2D BEM elasticity
}

\author{
Alberto Salvadori*,† \\ Department of Civil Engineering, University of Brescia, Via Branze 38, 25123 Brescia, Italy
}

\begin{abstract}
SUMMARY
In the context of two-dimensional linear elasticity, this paper presents the closed form of the integrals that arise from both the standard (collocation) boundary element method and the symmetric Galerkin boundary element method. Adopting polynomial shape functions of arbitrary degree on straight elements, finite part of Hadamard, Cauchy principal values and Lebesgue integrals are computed analytically, working in a local coordinate system. For the symmetric Galerkin boundary element method, a study on the singularity of the external integral is conducted and the outer weakly singular integral is analytically performed. Numerical tests are presented as a validation of the obtained results. Copyright (c) 2001 John Wiley \& Sons, Ltd.
\end{abstract}

KEY WORDS: integral equations; boundary element methods; analytical integrations; Hadamard's finite part; Cauchy's principal value

\section{INTRODUCTION}

In the framework of linear elasticity problems modelled by means of boundary integral equations (BIEs) [1], the present work concerns analytical integrations on polygonal domains in $\mathbf{R}^{2}$. As a reference problem, a homogeneous solid with polygonal domain $\Omega \subset \mathbf{R}^{2}$ and with boundary $\Gamma=\Gamma_{u} \cup \Gamma_{p}$ is considered. Assuming small strains and displacements, consider the following quasi-static external actions: tractions $\overline{\mathbf{p}}(\mathbf{x})$ on $\Gamma_{p}$, displacements $\overline{\mathbf{u}}(\mathbf{x})$ on $\Gamma_{u}$ and domain forces $\overline{\mathbf{f}}(\mathbf{x})$ in $\Omega$; the constitutive law is assumed to be isotropically linear elastic. The assumed problem is a prototype of a broad class of engineering applications. In fact, BIEs of linear elasticity, can be easily arranged to describe various engineering problems, among others: potential problems [2]; fracture mechanics problems [3], with internal pressure [4] and frictional contact [5]; multidomain problems [6,7], even in the case of non-linear interfaces [8]; steady Stokes flow of an incompressible newtonian fluid [9].

\footnotetext{
*Correspondence to: Alberto Salvadori, Department of Civil Engineering, University of Brescia, Via Branze 38, 25123 Brescia, Italy
}

†E-mail: alberto@ing.unibs.it

Copyright (c) 2001 John Wiley \& Sons, Ltd.

Received 28 January 2000

Revised 9 April 2001 
The boundary integral formulation of a linear elastic problem was proposed by Rizzo [10], stemming from Somigliana's identity, which is the boundary integral representation of displacements in the interior of the domain $\mathbf{x} \in \Omega$. Somigliana's identity is based on Green's functions (also called kernels) which represent components $u_{i}$ of the displacement vector $\mathbf{~}$ in a point $\mathbf{x}$ due to: (i) a unit force concentrated in space (point $\mathbf{y}$ ) and acting on the unbounded elastic space $\Omega_{\infty}$ in direction $j$ (such functions are gathered in matrix $\mathbf{G}_{u u}(\mathbf{x}-\mathbf{y})$ ); (ii) a unit relative displacement concentrated in space (at a point $\mathbf{y}$ ), crossing a surface with normal $\mathbf{l}(\mathbf{y})$ and acting on the unbounded elastic space $\Omega_{\infty}$ (in direction $j$ ) (gathered in matrix $\mathbf{G}_{u p}(\mathbf{x}-\mathbf{y})$ ) (Appendix A).

Since all the above-introduced kernels are infinitely smooth in their domain, which is the whole space $\mathbf{R}^{2}$ with exception of the origin (that is $\mathbf{x} \neq \mathbf{y}$ ), the traction operator can be applied to Somigliana's identity, thus obtaining the boundary integral representation of tractions on a surface of normal $\mathbf{n}(\mathbf{x})$ in the interior of the domain [11]. Such representation formula (by some authors named 'hypersingular identity' [12]) involves Green's functions (collected in matrices $\mathbf{G}_{p u}$ and $\mathbf{G}_{p p}$ ) which describe components $\left(p_{i}\right)$ of the traction vector $\mathbf{p}$ on a surface of normal $\mathbf{n}(\mathbf{x})$ due to: (i) a unit force concentrated in space (point $\mathbf{y}$ ) and acting on the unbounded elastic space $\Omega_{\infty}$ in direction $j$; (ii) a unit relative displacement concentrated in space (at a point $\mathbf{y}$ ), crossing a surface with normal $\mathbf{l}(\mathbf{y})$ and acting on the unbounded elastic space $\Omega_{\infty}$ (in direction $j$ ).

BIEs for the linear elastic problem can be derived from the aforementioned two representation formulae performing the boundary limit $\Omega \ni \mathbf{x} \rightarrow \mathbf{x}^{0} \in \Gamma$. For the hypersingular identity, the boundary limit must be considered at a smooth point $\mathbf{x}^{\mathrm{o}}$ with a well-defined normal vector $\mathbf{n}\left(\mathbf{x}^{0}\right)$ [13]. The two integral equations, usually referred to as displacements and traction equations, are also called 'dual' BIEs [14].

In the limit process, singularities of Green's functions are triggered off. Kernel $\mathbf{G}_{u u}$ shows an integrable singularity (named 'weak') $O\left(r^{-1}\right)$; kernels $\mathbf{G}_{u p}$ and $\mathbf{G}_{p u}$ present a strong singularity of $O\left(r^{-2}\right)$; kernel $\mathbf{G}_{p p}$ is usually named hypersingular, since it shows a singularity of $O\left(r^{-3}\right)$ greater than the dimension of the integral [15].

By the approach of [16], all singular terms cancel out in the limit process (and without recourse to any a-priori interpretation in the finite part sense). However, there exists an intimate relationship between hypersingular BIEs and finite part integrals (HFPs) in the sense of Hadamard [17]. In References [15, 18] among others, it has been proved that a hypersingular integral can be interpreted as a HFP in the limit as an internal point source approaches the boundary. In Reference [19], the same conclusion has been obtained by an alternate definition of HFP, without the need for a limiting process. Making recourse to the distribution theory, in Reference [14] the dual BIEs are obtained by the application of a trace operator to the representation formulae. In such an approach, the strongly singular and hypersingular integrals can be expressed by means of discontinuity jumps (also named as 'free terms') of these integrals on the boundary summed with the values of the integrals on the boundary existing only in the sense of the Cauchy principal value (CPV) or in the sense of the HFP. By exploiting Green's functions properties, the commutativity of the two operations of traction and trace has also been proved, showing the consistency of all different approaches of derivations of the BIEs.

As mentioned above, strongly singular kernels $\mathbf{G}_{u p}$ and $\mathbf{G}_{p u}$ generate free terms, $\mathbf{C}\left(\mathbf{x}^{\mathrm{o}}\right)$ and $\mathbf{D}\left(\mathbf{x}^{\mathrm{o}}\right)$, respectively, that hold $\frac{1}{2} \mathbf{I}$ for smooth boundaries $\left.[13,20,21]\right)$ in the limit process. It has been shown [16] that the hypersingular kernel generates a free term when the boundary 
curvature and the tangent vector to the boundary are not smooth. Discarding these conditions, the BIEs of the linear elastic problem read as follows [14]:

$$
\begin{aligned}
& \mathbf{C}(\mathbf{x}) \mathbf{u}(\mathbf{x})+\int_{\Gamma_{p}} \mathbf{G}_{u p}(\mathbf{r} ; \mathbf{l}(\mathbf{y})) \mathbf{u}(\mathbf{y}) \mathrm{d} \mathrm{d} \mathbf{y}+\int_{\Gamma_{u}} \mathbf{G}_{u p}(\mathbf{r} ; \mathbf{l}(\mathbf{y})) \overline{\mathbf{u}}(\mathbf{y}) \mathrm{d} \mathbf{y} \\
& \quad=\int_{\Gamma_{u}} \mathbf{G}_{u u}(\mathbf{r}) \mathbf{p}(\mathbf{y}) \mathrm{d} \mathbf{y}+\int_{\Gamma_{p}} \mathbf{G}_{u u}(\mathbf{r}) \overline{\mathbf{p}}(\mathbf{y}) \mathrm{d} \mathbf{y}+\int_{\Omega} \mathbf{G}_{u u}(\mathbf{r}) \overline{\mathbf{f}}(\mathbf{y}) \mathrm{d} \mathbf{y}, \quad \mathbf{x} \in \Gamma \\
& \mathbf{D}(\mathbf{x}) \mathbf{p}(\mathbf{x})+f_{\Gamma_{p}} \mathbf{G}_{p p}(\mathbf{r} ; \mathbf{n}(\mathbf{x}) ; \mathbf{l}(\mathbf{y})) \mathbf{u}(\mathbf{y}) \mathrm{d} \mathbf{y}+f_{\Gamma_{u}} \mathbf{G}_{p p}(\mathbf{r} ; \mathbf{n}(\mathbf{x}) ; \mathbf{l}(\mathbf{y})) \overline{\mathbf{u}}(\mathbf{y}) \mathrm{d} \mathbf{y} \\
& =f_{\Gamma_{u}} \mathbf{G}_{p u}(\mathbf{r} ; \mathbf{n}(\mathbf{x})) \mathbf{p}(\mathbf{y}) \mathrm{d} \mathbf{y}+\int_{\Gamma_{p}} \mathbf{G}_{p u}(\mathbf{r} ; \mathbf{n}(\mathbf{x})) \overline{\mathbf{p}}(\mathbf{y}) \mathrm{d} \mathbf{y}+\int_{\Omega} \mathbf{G}_{p u}(\mathbf{r} ; \mathbf{n}(\mathbf{x})) \overline{\mathbf{f}}(\mathbf{y}) \mathrm{d} \mathbf{y}, \quad \mathbf{x} \in \Gamma
\end{aligned}
$$

having set $\mathbf{r}=\mathbf{x}-\mathbf{y}$. After imposing the fulfillment of Equation (1) on $\Gamma_{u}$ and of Equation (2) on $\Gamma_{p}$, one obtains a linear boundary integral problem whose operator is symmetric with respect to the classical bilinear form (see [22-25]). Accordingly, the problem admits of a variational formulation [26, 27] and its solution is shown to be a saddle-point of a given functional $\Psi(\mathbf{u}, \mathbf{p})$, whose expression can be found for instance in Reference [28]. Energy implications have been largely investigated (see [29] among others).

Let $\hat{\mathbf{u}}(\mathbf{y}), \hat{\mathbf{p}}(\mathbf{y})$ be discrete approximations of the displacement field $\mathbf{u}(\mathbf{y})$ and of the traction field $\mathbf{p}(\mathbf{y})$, respectively. The discretization of the unknown fields permits to transform the BIEs into sets of algebraic equations. Two main techniques have been successfully developed to this aim: the collocation [2] and the symmetric Galerkin [30] methods (SGBEM).

The collocation approach requires the fulfillment of the displacement equation onto a selected set of collocation points, $\mathbf{x}_{i}^{*} \in \Gamma$, after having substituted $\mathbf{u}(\mathbf{y})$ and $\mathbf{p}(\mathbf{y})$ with the discrete approximations $\hat{\mathbf{u}}(\mathbf{y}), \hat{\mathbf{p}}(\mathbf{y})$. In this technique, one has to deal with integrals of the following form:

$$
\int_{\Gamma_{s}} \mathbf{G}_{r s}\left(\mathbf{x}_{i}^{*}-\mathbf{y}\right) \psi_{h}(\mathbf{y}) \mathrm{d} \Gamma(\mathbf{y}) \quad r=u, s=u, p
$$

denoting with $\psi_{h}(\mathbf{y})$ shape functions that model the unknown fields on the boundary.

After having substituted $\mathbf{u}(\mathbf{y})$ and $\mathbf{p}(\mathbf{y})$ with the discrete approximations $\hat{\mathbf{u}}(\mathbf{y}), \hat{\mathbf{p}}(\mathbf{y})$ into the functional $\boldsymbol{\Psi}(\hat{\mathbf{u}}, \hat{\mathbf{p}})$, the SGBEM approach is obtained by performing the first variation with respect to the (discrete) unknowns. In this formulation, one has to deal with integrals of the following form:

$$
\int_{\Gamma_{r}} \psi_{k}(\mathbf{x}) \int_{\Gamma_{s}} \mathbf{G}_{r s}(\mathbf{x}-\mathbf{y}) \psi_{h}(\mathbf{y}) \mathrm{d} \Gamma(\mathbf{y}) \mathrm{d} \Gamma(\mathbf{x}), \quad r, s=u, p
$$

where $\psi_{k}(\mathbf{x}), \psi_{h}(\mathbf{y})$ are, respectively, test and shape functions that model the displacement and traction fields on the boundary. The evaluation of (3) and (4) is not a trivial task, because of the involved singularities, especially for the hypersingular kernel. Several techniques, 
collectable in three principal groups, have been proposed for their evaluation: (i) regularization techniques, (ii) numerical integrations, (iii) analytical integrations.

By a regularization procedure, the strongly singular and hypersingular integrals are analytically manipulated to convert them into, at most, weakly singular integrals, which can then be computed throughout different quadrature schemes. Regularization procedures have been obtained by means of simple solutions [31, 32]; by applying the Stokes theorem [18, 33]; via integration by parts [34-36].

Numerical methods for the evaluation of the CPV were proposed first in Reference [37]. There is nowadays an extensive literature on this subject (see, among others [38-44]). A huge amount of literature concerns the numerical evaluation of hypersingular integrals: among others $[15,18,19,38,45-47]$.

Analytical integrations have been basically performed towards two schemes. In the first scheme (see e.g. $[12,16,45,46,48]$ ), the source point is fixed, while the boundary around the source point is temporarily deformed to allow an analytical evaluation of contributions from singular kernels, and then the limit is taken as the deformed boundary shrinks back to the actual boundary. In the second approach, see among others $[40,49,50]$, the source point $\mathbf{x}$ is first moved away from the boundary; integrals are evaluated analytically and a limit process is then performed to bring the source point back to the boundary. In all the aforementioned papers, analytical integrations are provided for all singular integrals, while standard quadrature formulae are used for non-singular integrals. A complete analytical integration of 2D elastostatic kernels has been provided in Reference [51] for linear shape functions on polygonal domains, directly evaluating HFP and CPV.

The present note provides a further contribution in the context of analytical integrations. It is focused on the analytical evaluation of the single integration process pertaining to the collocation technique (3) and of the double integration process (4) pertaining to the SGBEM, when the domain is an open polygon. Discretization is here considered by means of straight elements adopting polynomial test and shape functions of arbitrary degree. This allows for a $h$ - as well as a $p$ - and $h-p$ approximation technique.

In Section 2, the problem is shortly formulated, making reference to [8] for further details. By introducing a local orthogonal coordinate system, an effective representation of the involved integrals is given. In Section 3, the analytical integration of (3) is carried out. The Cauchy principal values and Hadamard's finite parts are computed both directly and by a limit process. In this last case, well-known free terms $[16,18]$ naturally arise. The performed analytical integrations are exhaustive for every formulation that entails only one integration process, with exception of corners. In the frame of the Galerkin approach, the singularity analysis in the outer integration process is required and developed in Section 4. The outer integration process is proved to involve at most weak singularities; this property allows the use of numerical quadrature rules with high efficiency and accuracy $[52,53]$ that require large computational effort, though. As an alternative, computationally advantageous in some circumstances, the analytical integration of the weakly singular integrals is performed in Section 5. In Section 6, two applications are given to validate the proposed results. As first a Dirichlet problem on a disk is proposed, where the $p$-technique reveals to be very efficient both for the collocation and the SGBEM. Furthermore, a multizone problem with rigid interface is proposed and solved via the SGBEM. A pure integration test is also conducted, comparing analytical integration with recently proposed numerical integration schemes [42]. 


\section{PROBLEM FORMULATION}

Define test $\psi_{k}(\mathbf{x})$ and shape functions $\psi_{h}(\mathbf{y})$ in Equations (3) and (4) as follows. Let $\Gamma_{h}$ be a decomposition of the polygonal boundary $\Gamma$ with nodes $\left\{\mathbf{P}_{h}, h=1,2, \ldots, N_{h}\right\}$. Let $T_{j}$ be the generic segment of $\Gamma_{h}$. Chose over $T_{j}$ a local basis $\left\{\varphi_{0}, \varphi_{1}, \ldots, \varphi_{N_{j}}\right\}$. Here, $\varphi_{j}$ is a polynomial (usually lagrangian) of degree $N_{j}$ defined on a subset of $\left\{\mathbf{P}_{h}\right\}$ of $N_{j}+1$ nodes in $T_{j}$. Collect in set $\mathscr{T}_{h}=\left\{T_{j}\right\}_{j=1}^{N}$ the (two at most, i.e. $N \leqslant 2$ ) segments having the common vertex $\mathbf{P}_{h}$ (see Figure 1); then $\psi_{h}$ is defined as

$$
\psi_{h}(\mathbf{y}):= \begin{cases}\varphi_{n}(\mathbf{y}), & \mathbf{y} \in T_{j}, j=1, \ldots, N \\ 0 & \text { elsewhere }\end{cases}
$$

where the index $n$ selects the local basis function on $T_{j}$ such that $\varphi_{n}\left(\mathbf{P}_{h}\right)=1$. By construction, $\psi_{h}(\mathbf{y})$ is continuous over $\Gamma_{h}$, and its support coincides with $\mathscr{T}_{h}$.

The inner 'integral' in (4) takes the form

$$
\mathbf{F}_{r s}(\mathbf{x}):=\int_{\Gamma_{s}} \mathbf{G}_{r s}(\mathbf{r}) \psi_{h}(\mathbf{y}) \mathrm{d} \mathbf{y}=\int_{\operatorname{supp}\left(\psi_{h}\right)} \mathbf{G}_{r s}(\mathbf{r}) \psi_{h}(\mathbf{y}) \mathrm{d} \mathbf{y}=\sum_{j=1}^{N} \mathbf{F}_{r s}^{j}(\mathbf{x})
$$

having defined $\mathbf{r}=\mathbf{x}-\mathbf{y}$ and

$$
\mathbf{F}_{r s}^{j}(\mathbf{x}):=\int_{T_{j}} \mathbf{G}_{r s}(\mathbf{r}) \varphi_{n}(\mathbf{y}) \mathrm{d} \mathbf{y}
$$

A suitable choice of an orthogonal cartesian coordinate system (abbreviated as 'c.s.') allows an effective representation for $\varphi_{n}(\mathbf{y})$. The choice of an orthogonal c.s. is arbitrary because (4) is invariant in transforming the orthogonal cartesian c.s. for the inner variable $\mathbf{y}$ if Jacobian is unit and no distortions are introduced with regard to Hadamard's finite part [54].

Let $\mathscr{L} \equiv\left\{y_{1}, y_{2}\right\}$ define a local c.s. with origin in the midpoint of $T_{j}$, oriented as in Figure 2 . If $\mathbf{y} \in T_{j}$ then $\mathbf{l}(\mathbf{y})=(0,1), y_{2}=0,-l_{j} \leqslant y_{1} \leqslant l_{j}$ and $\varphi_{n}(\mathbf{y})=\mathbf{y}_{1}^{\mathrm{T}} \mathbf{a}_{n}$, having defined

$$
\mathbf{y}_{1}^{\mathrm{T}}:=\left\{1, y_{1}, y_{1}^{2}, \ldots, y_{1}^{N_{j}}\right\}, \quad \mathbf{a}_{n}^{\mathrm{T}}:=\left\{a_{n}^{(0)}, a_{n}^{(1)}, a_{n}^{(2)}, \ldots, a_{n}^{\left(N_{j}\right)}\right\}
$$

From the binomial expansion

$$
y_{1}^{i}=\left(x_{1}-r_{1}\right)^{i}=\sum_{k=0}^{i}\left(\begin{array}{c}
i \\
k
\end{array}\right)(-1)^{k} x_{1}^{(i-k)} r_{1}^{k}
$$

it is straightforward to get

$$
\mathbf{y}_{1}^{\mathrm{T}}=\mathbf{r}_{1}^{\mathrm{T}} \mathbf{X}
$$

where

$$
\begin{aligned}
\mathbf{r}_{1}^{\mathrm{T}} & :=\left\{1, r_{1}, r_{1}^{2}, \ldots, r_{1}^{N_{j}}\right\} \\
\mathbf{X}_{i j} & :=-(-1)^{i}\left(\begin{array}{c}
j-1 \\
i-1
\end{array}\right) x_{1}^{(j-i)} \quad i, j=1,2, \ldots, N_{j}+1
\end{aligned}
$$




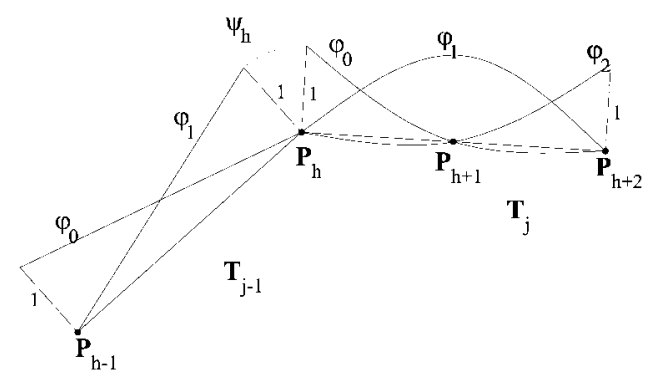

Figure 1. Local $\varphi_{n}(\mathbf{x})$ and global $\psi_{h}(\mathbf{x})$ shape function.

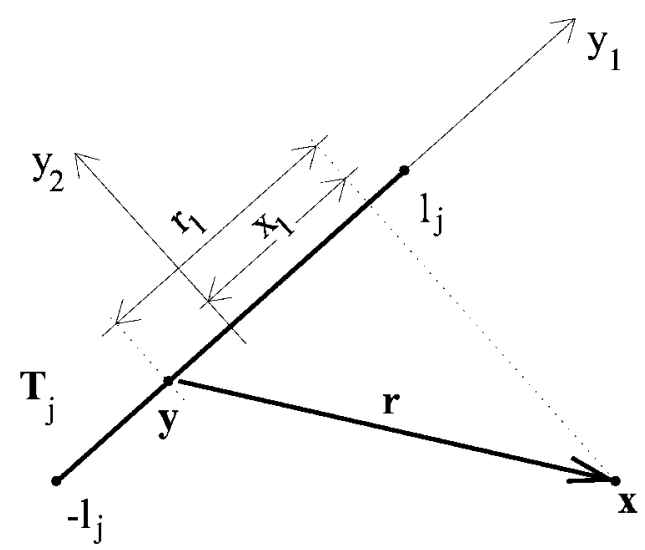

Figure 2. Local coordinate system $\mathscr{L}$.

Therefore, Equation (6) becomes

$$
\mathbf{F}_{r s}^{j}(\mathbf{x})=\left.\int_{x_{1}-l_{j}}^{x_{1}+l_{j}} \mathbf{G}_{r s}(\mathbf{r})\left(\begin{array}{cc}
\mathbf{r}_{1}^{\mathrm{T}} & 0 \\
0 & \mathbf{r}_{1}^{\mathrm{T}}
\end{array}\right) \mathrm{d} r_{1}\right|_{r_{2}=x_{2}}\left(\begin{array}{cc}
\mathbf{X} \mathbf{a}_{n} & 0 \\
0 & \mathbf{X} \mathbf{a}_{n}
\end{array}\right)
$$

In conclusion, to perform the inner integral in the local c.s. $\mathscr{L}$ one only needs to evaluate the following one

$$
\mathbf{K}_{r s}^{j}(\mathbf{x}):=\left.\int_{x_{1}-l_{j}}^{x_{1}+l_{j}} \mathbf{G}_{r s}(\mathbf{r})\left(\begin{array}{cc}
\mathbf{r}_{1}^{\mathrm{T}} & 0 \\
0 & \mathbf{r}_{1}^{\mathrm{T}}
\end{array}\right) \mathrm{d} r_{1}\right|_{r_{2}=x_{2}}
$$

which depends on the kernel $\mathbf{G}_{r s}$ and on the position of the point $\mathbf{x}$ with respect to $T_{j}$. Kernels $\mathbf{G}_{u u}(\mathbf{x}-\mathbf{y}), \mathbf{G}_{u p}(\mathbf{x}-\mathbf{y}, \mathbf{l}(\mathbf{y})), \mathbf{G}_{p u}(\mathbf{x}-\mathbf{y}, \mathbf{n}(\mathbf{x}))$ and $\mathbf{G}_{p p}(\mathbf{x}-\mathbf{y}, \mathbf{n}(\mathbf{x}), \mathbf{l}(\mathbf{y}))$ are singular with respect to $\mathbf{y}$ depending on the position of $\mathbf{x}$ with respect to $T_{j}$. We will therefore discuss separately the item $\mathbf{x} \notin \bar{T}_{j}$ that leads to a classic inner integral, from item $\mathbf{x} \in T_{j}$ that leads to a finite part of Hadamard. 


\section{INNER ANALYTICAL INTEGRATION}

\subsection{Lebesgue integrals}

Easy algebraic manipulations (see [8]) lead from integral (8) to the following basic integrals:

$$
\int_{x_{1}-l_{j}}^{x_{1}+l_{j}} r_{1}^{k} \log (r) \mathrm{d} r_{1} ; \quad \int_{x_{1}-l_{j}}^{x_{1}+l_{j}} \frac{r_{1}^{k}}{r^{2}} \mathrm{~d} r_{1} ; \quad \int_{x_{1}-l_{j}}^{x_{1}+l_{j}} \frac{r_{1}^{k}}{r^{4}} \mathrm{~d} r_{1} ; \int_{x_{1}-l_{j}}^{x_{1}+l_{j}} \frac{r_{1}^{k}}{r^{6}} \mathrm{~d} r_{1}
$$

that have been analytically solved when $\mathbf{x} \notin \bar{T}_{i}$ (results in Appendix B). Collecting all common terms, the following compact expressions are the outcome for integrals (8) in the local coordinate system $\mathscr{L}$ :

$$
\begin{aligned}
& \mathbf{K}_{u u}^{j}(\mathbf{x})=\frac{1}{8 \pi} \frac{1}{G(1-v)}\left[\log \left(r^{2}\right) \mathbf{L}_{u u}+\arctan \left(\frac{r_{1}}{x_{2}}\right) \mathbf{A}_{u u}+\mathbf{P}_{u u}\right]_{r_{1}=x_{1}-l_{j}}^{r_{1}=x_{1}+l_{j}} \\
& \mathbf{K}_{u p}^{j}(\mathbf{x})=\frac{1}{4 \pi} \frac{1}{1-v}\left[\log \left(r^{2}\right) \mathbf{L}_{u p}+\arctan \left(\frac{r_{1}}{x_{2}}\right) \mathbf{A}_{u p}+\frac{1}{r^{2}} \mathbf{S}_{u p}+\mathbf{P}_{u p}\right]_{r_{1}=x_{1}-l_{j}}^{r_{1}=x_{1}+l_{j}} \\
& \mathbf{K}_{p u}^{j}(\mathbf{x})=\frac{1}{4 \pi} \frac{1}{1-v}\left[\log \left(r^{2}\right) \mathbf{L}_{p u}+\arctan \left(\frac{r_{1}}{x_{2}}\right) \mathbf{A}_{p u}+\frac{1}{r^{2}} \mathbf{S}_{p u}+\mathbf{P}_{p u}\right]_{r_{1}=x_{1}-l_{j}}^{r_{1}=x_{1}+l_{j}} \\
& \mathbf{K}_{p p}^{j}(\mathbf{x})=\frac{G}{4 \pi} \frac{1}{1-v}\left[\log \left(r^{2}\right) \mathbf{L}_{p p}+\arctan \left(\frac{r_{1}}{x_{2}}\right) \mathbf{A}_{p p}+\frac{1}{r^{2}} \mathbf{S}_{p p}+\frac{1}{r^{4}} \mathbf{H}_{p p}+\mathbf{P}_{p p}\right]_{r_{1}=x_{1}-l_{j}}^{r_{1}=x_{1}+l_{j}}
\end{aligned}
$$

where $r^{2}=r_{1}^{2}+x_{2}^{2}$ and $\mathbf{L}_{u u}, \mathbf{A}_{u u}, \mathbf{P}_{u u}, \mathbf{L}_{u p}, \mathbf{A}_{u p}, \mathbf{S}_{u p}, \mathbf{P}_{u p}, \mathbf{L}_{p u}, \mathbf{A}_{p u}, \mathbf{S}_{p u}, \mathbf{P}_{p u}, \mathbf{L}_{p p}, \mathbf{A}_{p p}, \mathbf{S}_{p p}, \mathbf{H}_{p p}$ and $\mathbf{P}_{p p}$ are suitable matrices. Their expressions have been evaluated by means of the commercial code MATHEMATICA 4.0 for polynomial shape functions of arbitrary degree. One finds in Reference [8] their expression up to degree 5 while, for shortage of space, results relevant to quadratic shape functions are collected in Appendix C.

As a particular instance of $\mathbf{x} \notin T_{i}$, consider $x_{2}=0$ (see Figure 3 ). The expression of the hypersingular kernel $\mathbf{G}_{p p}$ simplifies being $x_{2}=0$ and $\mathbf{r} \cdot \mathbf{l}=0$.

$$
\mathbf{G}_{p p}(\mathbf{x}-\mathbf{y}, \mathbf{n}(\mathbf{x}), \mathbf{l}(\mathbf{y}))=\frac{G}{2 \pi} \frac{1}{1-v}\left(\begin{array}{ll}
n_{2} & n_{1} \\
n_{1} & n_{2}
\end{array}\right) \frac{1}{r_{1}^{2}}
$$

Equation (12) specifies as

$$
\mathbf{K}_{p p}^{j}(\mathbf{x})=\frac{G}{2 \pi} \frac{1}{1-v}\left(\begin{array}{ll}
n_{2} & n_{1} \\
n_{1} & n_{2}
\end{array}\right)\left(\begin{array}{cc}
\mathbf{r}_{p p}(\mathbf{x}) & \mathbf{0} \\
\mathbf{0} & \mathbf{r}_{p p}(\mathbf{x})
\end{array}\right)
$$




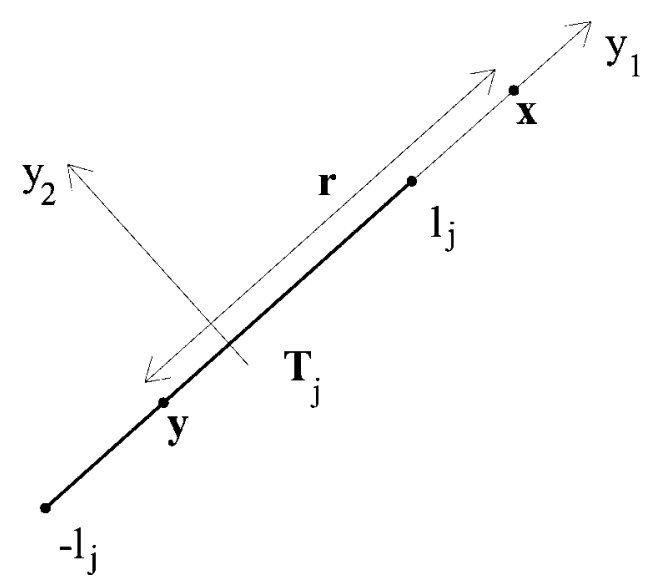

Figure 3. $\mathbf{x} \notin T_{i}$, but $x_{2}=0$.

having set

$$
\mathbf{r}_{p p}(\mathbf{x}):=\left.\left(-\frac{1}{r_{1}} ; \log \left|r_{1}\right| ; \cdots \frac{r_{1}^{k-1}}{k-1} ; \cdots\right)\right|_{r_{1}=x_{1}-l_{j}} ^{r_{1}=x_{1}+l_{j}}
$$

Again in the case of $x_{2}=0$, the expression of the strongly singular kernel $\mathbf{G}_{p u}$ simplifies as

$$
\mathbf{G}_{p u}(\mathbf{x}-\mathbf{y}, \mathbf{n}(\mathbf{x}))=-\frac{1}{4 \pi} \frac{1}{1-v}\left(\begin{array}{cc}
(3-2 v) n_{1} & (1-2 v) n_{2} \\
-(1-2 v) n_{2} & (1-2 v) n_{1}
\end{array}\right) \frac{1}{r_{1}}
$$

When $\mathbf{x} \notin T_{i}$, Equation (11) turns out to be

$$
\mathbf{K}_{p u}^{j}(\mathbf{x})=-\frac{1}{4 \pi} \frac{1}{1-v}\left(\begin{array}{cc}
(3-2 v) n_{1} & (1-2 v) n_{2} \\
-(1-2 v) n_{2} & (1-2 v) n_{1}
\end{array}\right)\left(\begin{array}{cc}
\mathbf{r}_{p u}(\mathbf{x}) & \mathbf{0} \\
\mathbf{0} & \mathbf{r}_{p u}(\mathbf{x})
\end{array}\right)
$$

having set

$$
\mathbf{r}_{p u}(\mathbf{x}):=\left.\left(\log \left|r_{1}\right| ; \cdots \frac{r_{1}^{k-1}}{k-1} ; \cdots\right)\right|_{r_{1}=x_{1}-l_{j}} ^{r_{1}=x_{1}+l_{j}}
$$

Similarly, it holds

$$
\mathbf{G}_{u p}(\mathbf{x}-\mathbf{y}, \mathbf{l}(\mathbf{y}))=-\frac{1}{4 \pi} \frac{1-2 v}{1-v}\left(\begin{array}{cc}
0 & 1 \\
-1 & 0
\end{array}\right) \frac{1}{r_{1}}
$$

and Equation (10) holds

$$
\mathbf{K}_{u p}^{j}(\mathbf{x})=-\frac{1}{4 \pi} \frac{1-2 v}{1-v}\left(\begin{array}{cc}
\mathbf{0} & \mathbf{r}_{p u}(\mathbf{x}) \\
-\mathbf{r}_{p u}(\mathbf{x}) & \mathbf{0}
\end{array}\right)
$$




\subsection{Hadamard's finite part}

Definition 1. Let $\varepsilon \rightarrow I(\varepsilon)$ denote a complex-valued function which is continuous in $\left(0, \varepsilon_{0}\right)$ and assume that

$$
I(\varepsilon)=I_{0}+I_{1} \log (\varepsilon)+\sum_{j=2}^{m} I_{j} \varepsilon^{1-j}+o(1) ; \quad \varepsilon \rightarrow 0
$$

where $I_{j} \in C$. Then $I_{0}$ is called the finite part of $I(\varepsilon)$.

In dealing with integrals, the finite part $I_{0}$ of a (usually) divergent integral $\int_{-\infty}^{+\infty} \phi(t) \mathrm{d} t$ is denoted by the symbol $f_{-\infty}^{+\infty} \phi(t) \mathrm{d} t$.

Consider

$$
I(\varepsilon):=\int_{-l_{j}}^{x_{1}-\varepsilon} \frac{1}{r_{1}^{2}} \mathbf{r}_{1}^{\mathrm{T}} \mathrm{d} y_{1}=\left(-\left.\frac{1}{r_{1}}\right|_{x_{1}-l_{j}} ^{-\varepsilon} ; \log \left|r_{1}\right|_{x_{1}-l_{j}}^{-\varepsilon} ;\left.\cdots \frac{r_{1}^{k-1}}{k-1}\right|_{x_{1}-l_{j}} ^{-\varepsilon} ; \cdots\right)
$$

By means of definition 1 and making use of the additive property of the finite part of Hadamard, one obtains

$$
f_{-l_{j}}^{l_{j}} \frac{1}{r_{1}^{2}} \mathbf{r}_{1}^{\mathrm{T}} \mathrm{d} y_{1}=\mathbf{r}_{p p}(\mathbf{x})
$$

with $\mathbf{r}_{p p}(\mathbf{x})$ defined in Equation (15). By means of (21) and setting $\mathbf{n}(\mathbf{x})=(0,1)$ in Equation (13) one obtains the following expression of the finite part of Hadamard of the integral (8), when $\mathbf{x} \in T_{j}$

$$
\mathbf{K}_{p p}^{j}(\mathbf{x})=\frac{G}{2 \pi} \frac{1}{1-v}\left(\begin{array}{cc}
\mathbf{r}_{p p}(\mathbf{x}) & \mathbf{0} \\
\mathbf{0} & \mathbf{r}_{p p}(\mathbf{x})
\end{array}\right)
$$

As a different approach, perform the limit process $\mathbf{K}_{p p}^{j}(\mathbf{z})$ s.t. $T_{j} \ngtr \mathbf{z} \rightarrow \mathbf{x} \in T_{j}$. By taking $x_{2} \rightarrow 0^{-}$, it holds

$$
\begin{aligned}
\lim _{x_{2} \rightarrow 0^{-}} \mathbf{L}_{p p} & =\frac{G}{4 \pi(1-v)}\left[\begin{array}{ccccc|ccccc}
0 & 1 & 0 & \ldots & 0 & 0 & 0 & 0 & \ldots & 0 \\
\hline 0 & 0 & 0 & \ldots & 0 & 0 & 1 & 0 & \ldots & 0
\end{array}\right] \\
\lim _{x_{2} \rightarrow 0^{-}} \mathbf{A}_{p p} & =\mathbf{0} \\
\lim _{x_{2} \rightarrow 0^{-}} \mathbf{S}_{p p} & =-\frac{G r_{1}}{2 \pi(1-v)}\left[\begin{array}{ccccc|ccccc}
1 & 0 & 0 & \ldots & 0 & 0 & 0 & 0 & \ldots & 0 \\
\hline 0 & 0 & 0 & \ldots & 0 & 1 & 0 & 0 & \ldots & 0
\end{array}\right] \\
\lim _{x_{2} \rightarrow 0^{-}} \mathbf{H}_{p p} & =\mathbf{0} \\
\lim _{x_{2} \rightarrow 0^{-}} \mathbf{P}_{p p}[1,1] & =\mathbf{P}_{p p}[2,2]=\frac{G}{2 \pi(1-v)}\left[\begin{array}{llllll}
0 & 0 & r_{1} & r_{1}^{2} / 2 & \ldots & r_{1}^{k} / k
\end{array}\right] \\
\lim _{x_{2} \rightarrow 0^{-}} \mathbf{P}_{p p}[1,2] & =\mathbf{P}_{p p}[2,1]=\mathbf{0}
\end{aligned}
$$

and Equation (22) is obtained immediately from (12). 
One concludes therefore that when $\mathbf{x} \notin \bar{T}_{j}$, the hyper singularities in integral (8) are not triggered off and $\mathbf{K}_{p p}^{j}(\mathbf{x})$ is a Lebesgue integral. On the contrary, as $\mathbf{x} \in T_{j}$ the 'integral' (8) does not exist in a Lebesgue sense but it can be seen in the finite part of Hadamard sense for the hypersingular kernel $\mathbf{G}_{p p}$. This property actually holds not only for (8) but for the traction boundary integral equation itself. Following the approach of [16], all singular terms cancel out in the limit process (and without recourse to any a-priori interpretation in the finite part sense). Although, there exists an intimate relationship between hypersingular boundary integral equations and finite part integrals in the sense of Hadamard: in References [15] and [18] among others, it has been proved that a hypersingular integral can be interpreted in the finite part sense in the limit process of an internal source point that approaches the boundary. In Reference [19], the same conclusion has been obtained by a different definition of finite part, without the need for any limit process.

Finally, it is worth stressing the equivalence between (14) and (22): this implies that when $x_{2}=0$, 'wherever $\mathbf{x}$ is placed (inside or outside $T_{i}$ )', $\mathbf{K}_{p p}^{j}$ assumes the same expression. In a distribution and in a more correct sense, we obtain that the function $\mathbf{K}_{p p}^{j}$ (not the value of $\mathbf{K}_{p p}^{j}(\mathbf{x})$ !) does not depend on $\mathbf{x}$.

\subsection{Cauchy principal value}

Definition 2. The CPV of the (usually) divergent integral $\int_{-\infty}^{+\infty}(\phi(t) / t) \mathrm{d} t$ is the finite quantity

$$
f_{-\infty}^{+\infty} \frac{\phi(t)}{t} \mathrm{~d} t:=\lim _{\varepsilon \rightarrow 0^{+}}\left(\int_{-\infty}^{-\varepsilon} \frac{\phi(t)}{t} \mathrm{~d} t+\int_{\varepsilon}^{+\infty} \frac{\phi(t)}{t} \mathrm{~d} t\right)
$$

Proposition. It is straightforward [55] to prove that

$$
f_{-\infty}^{+\infty} \frac{\phi(t)}{t} \mathrm{~d} t=f_{-\infty}^{0} \frac{\phi(t)}{t} \mathrm{~d} t+f_{0}^{+\infty} \frac{\phi(t)}{t} \mathrm{~d} t
$$

Property (23) permits to extend the concluding remarks of the previous section to the strongly singular kernels $\mathbf{G}_{u p}$ and $\mathbf{G}_{p u}$. In particular, it is possible to state that when $\mathbf{x} \in T_{j}$ the 'integral' (8) can be considered in the CPV sense [55] for the strongly singular kernels $\mathbf{G}_{u p}$ and $\mathbf{G}_{p u}$. The equivalence between the limit process approach and the CPV interpretation is proved in what follows.

From the Definition 1 and Property (23), one has

$$
f_{-l_{j}}^{l_{j}} \frac{1}{r_{1}} \mathbf{r}_{1}^{\mathrm{T}} \mathrm{d} y_{1}=\mathbf{r}_{p u}(\mathbf{x})
$$

and by setting $\mathbf{n}(\mathbf{x})=(0,1)$ in Equations (16) and (19) one obtains

$$
\mathbf{K}_{p u}^{j}(\mathbf{x})=\mathbf{K}_{u p}^{j}(\mathbf{x})=-\frac{1}{4 \pi} \frac{1-2 v}{1-v}\left(\begin{array}{cc}
\mathbf{0} & \mathbf{r}_{p u}(\mathbf{x}) \\
-\mathbf{r}_{p u}(\mathbf{x}) & \mathbf{0}
\end{array}\right)
$$


Through a limit process, by taking $x_{2} \rightarrow 0^{-}$in (11), one has

$$
\begin{aligned}
& \lim _{x_{2} \rightarrow 0^{-}} \mathbf{L}_{p u}=-\frac{1-2 v}{2}\left[\begin{array}{cccc|cccc}
0 & 0 & \ldots & 0 & 1 & 0 & \ldots & 0 \\
\hline-1 & 0 & \ldots & 0 & 0 & 0 & \ldots & 0
\end{array}\right] \\
& \lim _{x_{2} \rightarrow 0^{-}} \mathbf{A}_{p u}=-2(1-v)\left[\begin{array}{cccc|cccc}
1 & 0 & \ldots & 0 & 0 & 0 & \ldots & 0 \\
\hline 0 & 0 & \ldots & 0 & 1 & 0 & \ldots & 0
\end{array}\right] \\
& \lim _{x_{2} \rightarrow 0^{-}} \mathbf{S}_{p u}=\mathbf{0} \\
& \lim _{x_{2} \rightarrow 0^{-}} \mathbf{P}_{p u}[1,1]=\lim _{x_{2} \rightarrow 0^{-}} \mathbf{P}_{p u}[2,2]=\mathbf{0} \\
& \lim _{x_{2} \rightarrow 0^{-}} \mathbf{P}_{p u}[1,2]=-(1-2 v)\left[\begin{array}{lllll}
0 & r_{1} & r_{1}^{2} / 2 & \ldots & r_{1}^{k} / k
\end{array}\right] \\
& \lim _{x_{2} \rightarrow 0^{-}} \mathbf{P}_{p u}[2,1]=(1-2 v)\left[\begin{array}{lllll}
0 & r_{1} & r_{1}^{2} / 2 & \ldots & r_{1}^{k} / k
\end{array}\right]
\end{aligned}
$$

and Equation (11) becomes

$$
\mathbf{K}_{p u}^{j}(\mathbf{x})=-\frac{1}{4 \pi} \frac{1-2 v}{1-v}\left(\begin{array}{cc}
\mathbf{0} & \mathbf{r}_{p u}(\mathbf{x}) \\
-\mathbf{r}_{p u}(\mathbf{x}) & \mathbf{0}
\end{array}\right)+\left.\frac{1}{4}\left[\begin{array}{lll|lll}
1 & 0 & \ldots & 0 & 0 & \ldots \\
0 & 0 & \ldots & 1 & 0 & \ldots
\end{array}\right] \operatorname{sgn}\left(r_{1}\right)\right|_{r_{1}=x_{1}-l_{j}} ^{r_{1}=x_{1}+l_{j}}
$$

If $x_{1}<-l_{j}$ or $x_{1}>l_{j}$ then $\left.\operatorname{sgn}\left(r_{1}\right)\right|_{r_{1}=x_{1}-l_{j}} ^{r_{1}=x_{1}+l_{j}}=0$ so that (26) coincides with (17). On the contrary, when $-l_{j}<x_{1}<l_{j}$, it holds $\left.\operatorname{sgn}\left(r_{1}\right)\right|_{r_{1}=x_{1}-l_{j}} ^{r_{1}=x_{1}+l_{j}}=2$ and the second addend in Equation (26) turns out to be

$$
\frac{1}{2}\left[\begin{array}{llll|llll}
1 & 0 & \ldots & 0 & 0 & 0 & \ldots & 0 \\
\hline 0 & 0 & \ldots & 0 & 1 & 0 & \ldots & 0
\end{array}\right]
$$

Substituting (27) into (7) gives the discrete counterpart of the free-term for smooth boundaries

$$
\frac{1}{2}\left[\begin{array}{cccc|cccc}
1 & 0 & \ldots & 0 & 0 & 0 & \ldots & 0 \\
\hline 0 & 0 & \ldots & 0 & 1 & 0 & \ldots & 0
\end{array}\right]\left(\begin{array}{cc}
\mathbf{X} \mathbf{a}_{n} & 0 \\
0 & \mathbf{X a}_{n}
\end{array}\right)=\frac{1}{2} \varphi_{n}(\mathbf{x}) \mathbf{I}
$$

\section{OUTER INTEGRATION SINGULARITY ANALYSIS}

In this section, it will be proved that the external integral in Equation (4)

$$
\int_{\Gamma_{p}} \psi_{k}(\mathbf{x}) \sum_{j=1}^{N} \mathbf{K}_{p p}^{j}(\mathbf{x})\left(\begin{array}{cc}
\mathbf{X}(\mathbf{x}) \mathbf{a}_{n} & 0 \\
0 & \mathbf{X}(\mathbf{x}) \mathbf{a}_{n}
\end{array}\right) \mathrm{d} \mathbf{x}
$$

has a meaning in a Lebesgue sense even for the hypersingular kernel. For the sake of simplicity, suppose $\mathbf{x} \in T_{1} \subset \operatorname{supp}\left(\psi_{k}\right)$ and use the following notation: (i) take as the absolute 
coordinate system the local reference on the element $T_{1}$ and denote it by $\mathscr{R}$; (ii) denote by $\mathbf{x}^{(\mathrm{j})}$ the field point $\mathbf{x}$ when it is expressed in the local coordinate system $\mathscr{L}$ on the generic element $T_{j}$; (iii) omit the apex ${ }^{(1)}$ when $\mathbf{x}$ is expressed in $\mathscr{R}$, i.e. $\mathbf{x} \equiv \mathbf{x}^{(1)}$; (iv) take $\psi_{k}(\mathbf{x})$ and $\psi_{h}(\mathbf{x})$ of Section 2 as the Lagrangian test and shape functions.

Consider as first the 'easy' case of $\operatorname{supp}\left(\psi_{h}\right) \equiv T_{1}$. Equation (22) becomes:

$$
\mathbf{K}_{p p}^{1}(\mathbf{x})=\frac{G}{2 \pi} \frac{1}{1-v}\left(\begin{array}{cc}
\mathbf{r}_{p p}(\mathbf{x}) & \mathbf{0} \\
\mathbf{0} & \mathbf{r}_{p p}(\mathbf{x})
\end{array}\right)
$$

From the Definition (15) of $\mathbf{r}_{p p}(\mathbf{x})$, it is clear that all terms in Equation (29) are Lebesgue integrable with respect to $x_{1}$, except

$$
\mathbf{r}_{p p}(\mathbf{x})\left(\mathbf{e}_{1} \otimes \mathbf{e}_{1}\right) \mathbf{X} \mathbf{a}_{n}=-\left.\frac{1}{r_{1}}\right|_{r_{1}=x_{1}-l_{1}} ^{r_{1}=x_{1}+l_{1}} \varphi_{n}\left(x_{1}\right)
$$

As a consequence, the terms that 'can be singular' in (28) are the following:

$$
\frac{G}{2 \pi} \frac{\varphi_{n}\left(l_{1}\right)}{1-v} \int_{T_{1}} \psi_{k}(\mathbf{x}) \frac{1}{x_{1}-l_{1}} \mathrm{~d} x_{1} \mathbf{I}, \quad-\frac{G}{2 \pi} \frac{\varphi_{n}\left(-l_{1}\right)}{1-v} \int_{T_{1}} \psi_{k}(\mathbf{x}) \frac{1}{x_{1}+l_{1}} \mathrm{~d} x_{1} \mathbf{I}
$$

The hypothesis $\operatorname{supp}\left(\psi_{h}\right) \equiv T_{1}$ and the continuity of $\psi_{h}$ imply $\varphi_{n}\left(-l_{1}\right)=0, \varphi_{n}\left(l_{1}\right)=0$ and the 'integrability' of $\mathbf{F}_{p p}(\mathbf{x})$ immediately follows.

Consider now $\varphi_{n}\left(l_{1}\right)=1$. The choice of a lagrangian basis and the hypothesis of continuity require $\varphi_{n}\left(-l_{1}\right)=0$ and $T_{1} \subset \operatorname{supp}\left(\psi_{h}\right)$. Supposing $\operatorname{supp}\left(\psi_{h}\right)=T_{1} \cup T_{2}$ as in Figure 4, Equation

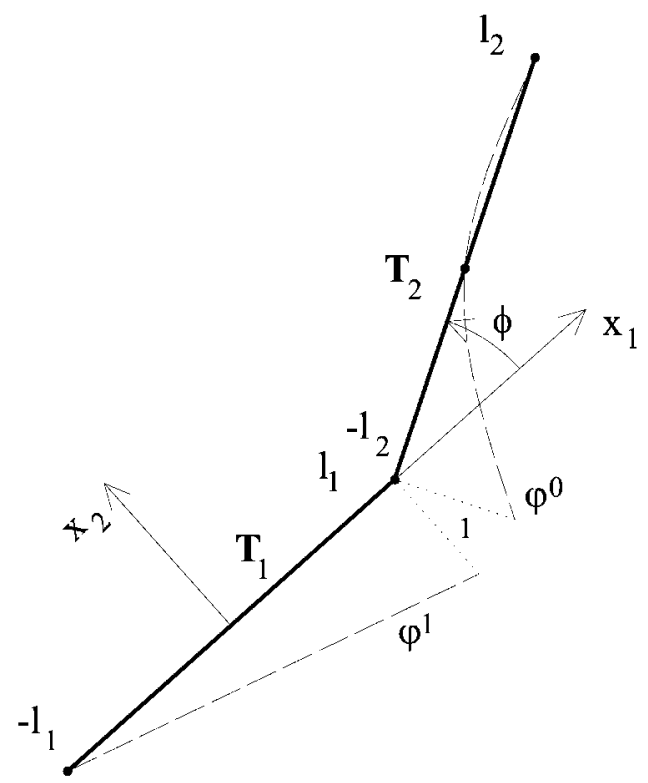

Figure 4. $\operatorname{supp}\left(\psi_{h}\right)=T_{1} \cup T_{2}$. 
(28) becomes

$$
\sum_{j=1}^{2} \int_{T_{j}} \psi_{k}(\mathbf{x}) \mathbf{K}_{p p}^{j}\left(\mathbf{x}^{(\mathrm{j})}\right)\left(\begin{array}{cc}
\mathbf{X}\left(\mathbf{x}^{(\mathrm{j})}\right) \mathbf{a}_{n} & 0 \\
0 & \mathbf{X}\left(\mathbf{x}^{(\mathrm{j})}\right) \mathbf{a}_{n}
\end{array}\right) \mathrm{d} \mathbf{x}
$$

About $\mathbf{K}_{p p}^{1}$, from Equation (30), singularities may arise only from the term

$$
\frac{G}{2 \pi} \frac{1}{1-v} \int_{T_{1}} \psi_{k}(\mathbf{x}) \frac{1}{x_{1}-l_{1}} \mathrm{~d} x_{1}
$$

About $\mathbf{K}_{p p}^{2}$, from identity (12) one notes that all terms are Lebesgue integral with exception of

$$
\left[\frac{1}{r^{2}} \mathbf{S}_{p p}+\frac{1}{r^{4}} \mathbf{H}_{p p}\right]^{r_{1}=x_{1}+l_{2}}\left(\begin{array}{cc}
\left(\mathbf{e}_{1} \otimes \mathbf{e}_{1}\right) \mathbf{X}^{(2)} \mathbf{a}_{n} & \mathbf{0} \\
\mathbf{0} & \left(\mathbf{e}_{1} \otimes \mathbf{e}_{1}\right) \mathbf{X}^{(2)} \mathbf{a}_{n}
\end{array}\right)
$$

In order to compare singularities, the factor (32) must be expressed in the absolute coordinate system $\mathscr{R}$. The reference change between the $\mathscr{L}$ on $T_{2}$ and $\mathscr{R}$ reads as

$$
\mathbf{x}^{(2)}=\mathbf{R}\left(\mathbf{x}-\mathbf{O}^{(2)}\right) ; \quad \mathbf{n}^{(2)}=\mathbf{R} \mathbf{n}
$$

having set

$$
\mathbf{R}=\left(\begin{array}{cc}
\cos \phi & -\sin \phi \\
\sin \phi & \cos \phi
\end{array}\right), \quad \mathbf{O}^{(2)}=\left(\begin{array}{c}
l_{1}+l_{2} \cos \phi \\
l_{2} \sin \phi
\end{array}\right)
$$

By means of (33), factor (32) becomes

$$
-\frac{1}{2 \pi} \frac{G}{1-v} \varphi_{n}\left(-l_{2}\right) \frac{1}{x_{1}-l_{1}} \mathbf{I}
$$

By noting that $\varphi_{n}\left(-l_{2}\right)=1$ for the continuity of $\psi_{h}(\mathbf{x})$, one finally states that $\mathbf{K}_{p p}^{2}$ generates the following singular integral:

$$
-\frac{1}{2 \pi} \frac{G}{1-v} \int_{T_{1}} \psi_{k}(\mathbf{x}) \frac{1}{x_{1}-l_{1}} \mathrm{~d} x_{1}
$$

It is so self shown that even if none of the two 'integrals'

$$
\int_{\Gamma_{p}} \psi_{k}(\mathbf{x}) \mathbf{F}_{p p}^{1}\left(\mathbf{x}^{(1)}\right) \mathrm{d} \mathbf{x}, \quad \int_{\Gamma_{p}} \psi_{k}(\mathbf{x}) \mathbf{F}_{p p}^{2}\left(\mathbf{x}^{(2)}\right) \mathrm{d} \mathbf{x}
$$

has a meaning in a Lebesgue sense, integral (28) is well defined.

\section{WEAKLY SINGULAR INTEGRALS}

In this section, it will be shown that all weakly singular integrals of (4) can be deduced from the following integral:

$$
\int z^{h} \log \left[z^{2}+c^{2}\right] \mathrm{d} z
$$


where $c \in \mathbf{R}, h \in \mathbf{N}$. Moreover, the following closed form can be proved by induction on $h$

$$
\begin{aligned}
\int z^{h} \log \left[z^{2}+c^{2}\right] \mathrm{d} z-C= & \frac{z^{h+1}}{h+1} \log \left[z^{2}+c^{2}\right] \\
& +\left(1-\mathrm{M}_{2}[h]\right)\left[(-1)^{h / 2} \frac{2 c^{h+1}}{h+1} \arctan \left(\frac{z}{c}\right)\right. \\
& \left.-\sum_{j=0}^{h / 2}(-1)^{(h-2 j) / 2} \frac{2 c^{h-2 j} z^{2 j+1}}{(h+1)(2 j+1)}\right] \\
& +\mathrm{M}_{2}[h]\left[(-1)^{(h-1) / 2} \frac{c^{h+1}}{h+1} \log \left(z^{2}+c^{2}\right)\right. \\
& \left.-\sum_{j=1}^{(h+1) / 2}(-1)^{h-j} \frac{c^{h+1-2 j} z^{2 j}}{(h+1) j}\right]
\end{aligned}
$$

where $C \in \mathbf{R}$ and $\mathrm{M}_{2}[h]$ stands for the remainder of the (integer) division $h \div 2$.

In order to prove that all weakly singular integrals of (4) can be deduced from (34), one defines test functions $\psi_{k}(\mathbf{x})$ as in Equation (5), denoting $\mathbf{x}_{1}:=\left\{1, x_{1}, x_{1}^{2}, \ldots, x_{1}^{N_{i}}\right\}, \mathbf{a}_{m}:=\left\{a_{m}^{(0)}\right.$, $\left.a_{m}^{(1)}, a_{m}^{(2)}, \ldots, a_{m}^{\left(N_{i}\right)}\right\}$ and $\varphi_{m}(\mathbf{x})=\mathbf{a}_{m}^{\mathrm{T}} \mathbf{x}_{1}$. Using Equations (7) and (8) integral (4) takes the following form for the generic kernel $\mathbf{G}_{r s}$ :

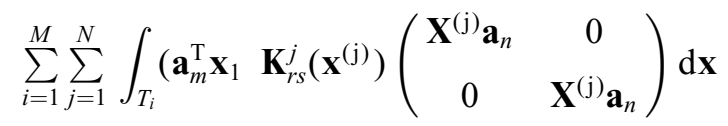

Here the apex ( $\mathrm{j})$ states that $\mathbf{x}$ is written in with respect to a local reference on $T_{j}$. With the aim of simplifying the notation, when not explicitly indicated by the apex $(j), \mathbf{x}$ will be expressed in the local reference on $T_{i}$ and the apex (i) omitted.

In view of identities (9)-(12), the external weakly singular integral in Equation (35) reads as

$$
\int_{-l_{i}}^{l_{i}}\left(\begin{array}{cc}
\mathbf{x}_{1} & 0 \\
0 & \mathbf{x}_{1}
\end{array}\right)\left[\log \left(r_{1}^{2}+\left(x_{2}^{(\mathrm{j})}\right)^{2}\right) \mathbf{L}_{r s}\right]_{r_{1}=x_{1}^{r_{1}}-x_{1}^{(\mathrm{j})}+l_{j}}^{r_{j}}\left(\begin{array}{cc}
\mathbf{X}^{(\mathrm{j})} & 0 \\
0 & \mathbf{X}^{(\mathrm{j})}
\end{array}\right) \mathrm{d} x_{1}
$$

After mapping $\mathbf{x}^{(\mathrm{j})}$ and $\mathbf{X}^{(\mathrm{j})}$ in the local reference on $T_{i}$ by means of a reference change, easy algebraic manipulations lead from Equation (36) to

$$
\left.\int_{-l_{i}}^{l_{i}}\left(\begin{array}{cc}
\mathbf{x}_{1} \otimes \mathbf{x}_{1} & 0 \\
0 & \mathbf{x}_{1} \otimes \mathbf{x}_{1}
\end{array}\right) \log \left[x_{1}^{2}+a(\eta) x_{1}+b(\eta)\right] \mathrm{d} x_{1} \boldsymbol{\Lambda}_{r s}(\eta)\right|_{\eta=-l_{j}} ^{\eta=l_{j}}
$$

where $\boldsymbol{\Lambda}_{r s}(\eta)$ is a suitable matrix, $a, b \in \mathbf{R}$ depends on $l_{j}$, with $b \geqslant 0$. As a conclusion, to perform the integration of the weakly singular part of (4), one has to solve the following 
integral:

$$
\int_{-l_{i}}^{l_{i}} x_{1}^{k} \log \left[x_{1}^{2}+a x_{1}+b\right] \mathrm{d} x_{1}=\sum_{h=0}^{k}\left(\begin{array}{l}
k \\
h
\end{array}\right)\left(-\frac{a}{2}\right)^{k-h} \int_{(a / 2)-l_{i}}^{(a / 2)+l_{i}} z^{h} \log \left[z^{2}+c^{2}\right] \mathrm{d} z
$$

where the following variable change has been used:

$$
z=x_{1}+a / 2 ; \quad c^{2}=b-a^{2} / 4
$$

\section{APPLICATIONS}

\subsection{A pure integration test}

As a test on the efficiency of the proposed formulae, a comparison with numerical integration rules proposed in References [42,43] has been performed. Comparisons can only give an overall criticism on the order of magnitude of computational efficiency, for they refer to different implementations using different languages and compilers.

The test refers to SGBEM, with reference to a regular polygon of 64 sides, leading to 256 degrees of freedom. Results are collected in Table I and refer to the evaluation of the whole matrix on a PentiumIII-350 Mhz personal computer.

For the analytical integrations implementation, most of the elapsed time is due to the external (exact) integration of weak singularities. Such an effort is computationally advantageous when one requires high precision in matrix entries, as seen in Table I.

On the other hand, when one sets a 'precision' tolerance in the matrix entries, computational effort of the analytical evaluation of weak singularities cannot be controlled. Setting a 'few digits precision' in the matrix entries implies the high time-consuming exact evaluation of weak singularities and thereafter a 'few digits precision' evaluation of all other non-singular terms, providing a global 'few digits precision' in the matrix entries. In such instances the implementation of specific numerical quadrature rules to control the outer weak singularities integration is surely suitable.

\subsection{An application}

Consider a disk of internal radius $a$ and external radius $b$, made of an elastic material $(E=2.06 e+05 \mathrm{MPa}, v=0.3)$ that undergoes an internal pressure $p$ and an external pressure $q$. The strain state of the disk is taken as plane, so that the analytical solution of the problem is well known [56].

Table I. Comparisons between analytical and numerical integration schemes.

\begin{tabular}{lccccc}
\hline \multicolumn{3}{c}{ Weakly singular kernel } & \multicolumn{2}{c}{ Hyper singular kernel } \\
\hline Precision & Analytical (s) & Numerical (s) & Precision & Analytical (s) & Numerical (s) \\
\hline 6 digits & 12.2 & 13.1 & 5 digits & 21.2 & 34.2 \\
11 digits & 15.5 & 20.8 & 11 digits & 40.4 & 98.3 \\
14 digits & 17.45 & 25.1 & 14 digits & 50.1 & Not reached \\
\hline
\end{tabular}




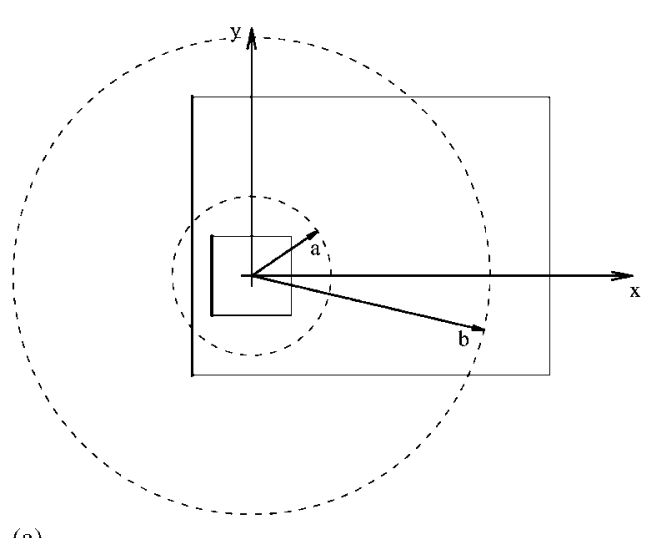

(a)

Figure 5. Problem geometry: (a) a disk and the selected geometry; and (b) dimensions $(a=40 \mathrm{~mm}, b=120 \mathrm{~mm})$

$A$ benchmark-If one takes $p=q$ the problem solution reads as follows:

$$
\mathbf{u}(\mathbf{x})=-\frac{v}{\lambda} p \mathbf{x}, \quad \sigma_{\theta}=\sigma_{r}=-p
$$

denoting with $\lambda$ the Lamè constant. By adopting at least linear polynomial shape functions for displacements, it is expected that the BEM approximation coincides with the analytical solution. In fact, the Galerkin and collocation schemes show an accuracy of more than 13 digits, i.e. a relative error lower than $10^{-13}$ on each term of the boundary approximation.

An example-If one takes $q=0$ the problem solution is no more polynomial with respect to $\mathbf{x}$ and reads as follows:

$$
\begin{aligned}
\mathbf{u}(\mathbf{x}) & =\frac{a^{2} p}{b^{2}-a^{2}}\left(\frac{v}{\lambda}+\frac{1+v}{E} \frac{b^{2}}{(\mathbf{x} \cdot \mathbf{x})}\right) \mathbf{x} \\
\sigma_{\theta}(\mathbf{x}) & =\frac{a^{2}}{(\mathbf{x} \cdot \mathbf{x})} \frac{(\mathbf{x} \cdot \mathbf{x})+b^{2}}{b^{2}-a^{2}} p, \quad \sigma_{r}(\mathbf{x})=\frac{a^{2}}{(\mathbf{x} \cdot \mathbf{x})} \frac{(\mathbf{x} \cdot \mathbf{x})-b^{2}}{b^{2}-a^{2}} p
\end{aligned}
$$

To avoid geometrical approximation of the disk by straight elements, solution (37) and (38) have been taken as a boundary condition on a different geometry, related to the disk as depicted in Figure 5(a). Dimensions are described in Figure 5(b), where $a=40 \mathrm{~mm}, b=120 \mathrm{~mm}$ have been adopted. The left side of the square plate and of the hole (depicted in Figure 5 by a thick line) are constrained imposing $\mathbf{u}(\mathbf{x})$ as in Equation (37). All other sides are loaded by means of traction forces due to the stress tensor corresponding to the displacement field (37) by the normal $\mathbf{n}(\mathbf{x})$.

The numerical approximation has been obtained via collocation and SGBEM through $h$ and $p$ techniques. As a starting point, a discretization made of 1 straight linear element for each side, corresponding to 8 global nodes and 16 global degrees of freedom (dof) has been considered. In the $h$-scheme, each side has been cut into half five times, up to a final 


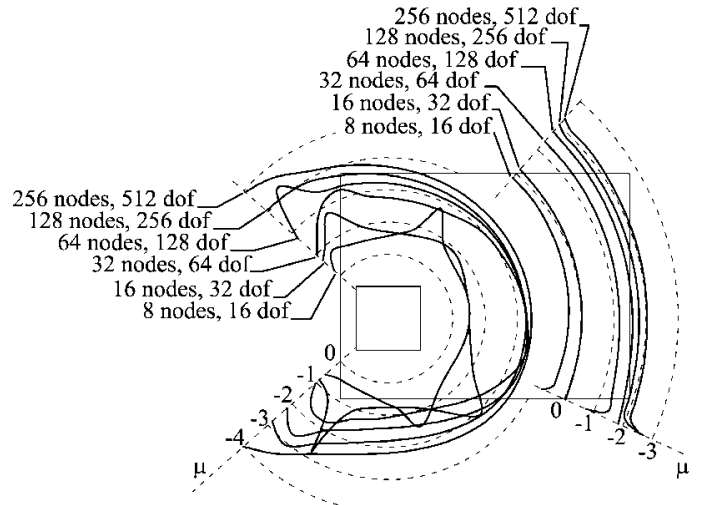

(a)

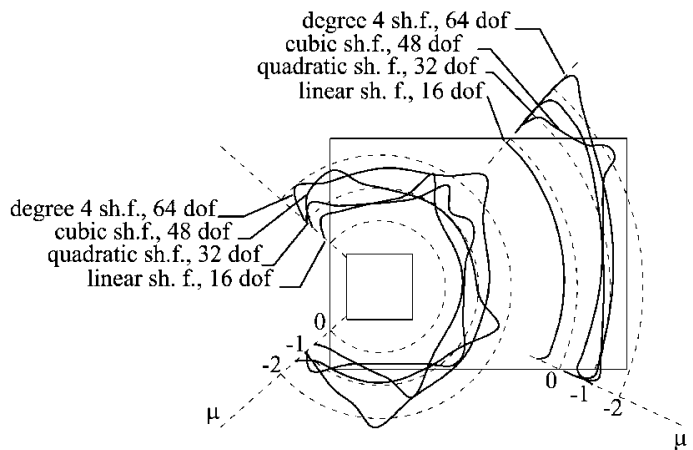

(b)

Figure 6. Displacement field error analysis: (a) $h$-technique; and (b) $p$-technique

discretization made of 256 global nodes and 512 global dof. In the $p$-scheme, the shape functions degree has been increased up to degree 4 and 64 dof.

Approximated displacement and stress fields are compared with analytical solutions (37) and (38) along the two circumferences of radius $a$ and $b$ (see Figure 5(a). Figure 6 concerns the radial displacement field. The relative error along the two circumferences of radius $a$ and $b$ (see Figure 5(a) is expressed by means of the variable

$$
\mu:=\log _{10}\left|\frac{\hat{u}_{r}(\mathbf{x})-u_{r}(\mathbf{x})}{u_{r}(\mathbf{x})}\right|
$$

Circumferences with $\mu=0$ correspond to the two circumferences of radius $a$ and $b$. With regard to the $h$-technique, one observes that precision increases close to the constrained side. This is due to the discretization, which is made of elements of smaller size in the shortest sides. The $p$-technique in fact, does not present a similar behaviour. About the $p$-technique, Figure 6(b) shows that the error plots are much less 'regular' than plots pertaining to the $h$-technique; increasing the shape function degree does not produce a better approximation everywhere. Comparing the two techniques, one notes that meshes with the same number of dof behaves similarly (see the two curves for 32 and 64 dof) around the hole; moreover, quadratic elements provide high benefit along the circumferences of radius $b$, compared with the $h$-curve for 32 dof.

Figure 7 concerns the radial stress field $\sigma_{r}(\mathbf{x})$. The relative error along the circumference of radius $a$ is expressed by means of the variable $\mu$ as in Equation (39). The absolute error along the circumference of radius $b$ is expressed by means of the variable

$$
\mu:=\log _{10}\left|\hat{\sigma}_{r}(\mathbf{x})\right|
$$

for being $\sigma_{r}(\mathbf{x})=0$ (see Equation (38)). Remarks made for the $h$-technique on the displacement field applies also to the stress field. On the contrary, the $p$-technique does not produce high benefits around the circumference of radius $b$, where the $h$-technique is preferable.

As a final remark, the numerical approximation is very good; in particular, the 512 dof mesh provides two exact significant digits in each considered point for both displacements 


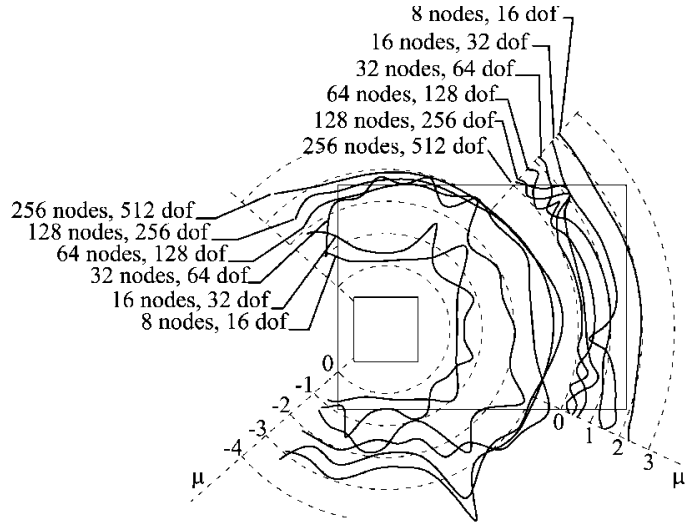

(a)

Figure 7. Stress field error analysis: (a) $h$-technique; and (b) $p$-technique

and stress. Furthermore, this example states that increasing the shape function degree can be effective together with a suitable mesh refinement ( $h p$-technique).

\section{CONCLUDING REMARKS}

In the context of 2D polygonal domains, the analytical integrations pertaining to the numerical approximation of boundary value problems formulated by means of integral equations have been presented for polynomial test and shape functions of arbitrary degree. The integration process has been performed both as a limit of the Lebesgue integral and directly in the CPV and Hadamard's finite parts sense.

With exception of corners, the analytical integrations performed in Equations (9)-(12) are exhaustive for every formulation that implies only one integration process. Typically, they totally solve integrals for the collocation BEM. Moreover, even in the frame of the Galerkin approach, the integrations needed to determine the internal distribution of displacements and stress (once the boundary nodal unknowns are given) are analytically achieved.

About the outer integration process, analytical integrations have been performed only for the weakly singular integrals, because the remaining integrals do not present any trouble in applying the usual Gaussian quadrature schemes. An object-oriented computer code has been developed that implements the proposed integration schemes. Approximation via $h-, p$ - and $h$ - $p$ techniques has been discussed in numerical applications; it shows an excellent agreement with the known analytical solutions.

The extension of the proposed analytical integrations to $3 \mathrm{D}$ problems is in progress [8]. Analytical integrations in 3D reveal to be much more complex and the analytical evaluation of the outer integral is under consideration with the aim of computational time saving.

Alternative strategies to implement the integration of the singular kernels arising in boundary element method are available in literature. Compared with the numerical integration of finite part [43], the proposed analytical integrations reveal to be low computational time consuming. A comparison with regularization techniques has not been carried out, yet. 
Besides computational efficiency, the availability of the closed form for the approximated displacement and stress fields entails the possibility of analytical manipulations which are hardly possible with alternative approaches. Typically, the possibility of finding the extremal values of the principal stress field is a major task in studying fracture initiation in unnotched structure. Furthermore, fracture propagation in bimaterial interfaces may have great advantages over the proposed analytical integrations. In these contexts, work is in progress.

\section{APPENDIX A: GREEN'S FUNCTIONS FOR LINEAR ELASTICITY}

Indicate with $\mathbf{r}=\mathbf{x}-\mathbf{y}$ and $r=\|\mathbf{r}\|$. Moreover, let $\mathbf{n}(\mathbf{x})$ denote the outward normal at the boundary $\Gamma$ at $\mathbf{x}$. Analogously, let $\mathbf{l}(\mathbf{y})$ denote the outward normal at the boundary $\Gamma$ at $\mathbf{y}$. Green's functions for linear elasticity are the following (see also [59]).

For plane strain the following expressions hold:

$$
\begin{aligned}
\mathbf{G}_{u u}(\mathbf{r})= & \frac{1}{8 \pi} \frac{1}{G(1-v)}\left(\frac{\mathbf{r} \otimes \mathbf{r}}{r^{2}}-(3-4 v) \log (r) \mathbf{I}\right) \\
\mathbf{G}_{p u}(\mathbf{r} ; \mathbf{n}(\mathbf{x}))= & \frac{1}{2 \pi} \frac{1}{(1-v)} \frac{1}{r^{2}}\left[(1-2 v)\left(\mathbf{S K W}(\mathbf{r} \otimes \mathbf{n})-\frac{\mathbf{r} \cdot \mathbf{n}}{2} \mathbf{I}\right)-(\mathbf{r} \cdot \mathbf{n}) \frac{\mathbf{r} \otimes \mathbf{r}}{r^{2}}\right] \\
\mathbf{G}_{u p}(\mathbf{r} ; \mathbf{l}(\mathbf{y}))= & \frac{1}{2 \pi} \frac{1}{(1-v)} \frac{1}{r^{2}}\left[(1-2 v)\left(\mathbf{S K W}(\mathbf{r} \otimes \mathbf{l})+\frac{\mathbf{r} \cdot \mathbf{l}}{2} \mathbf{I}\right)+(\mathbf{r} \cdot \mathbf{l}) \frac{\mathbf{r} \otimes \mathbf{r}}{r^{2}}\right] \\
\mathbf{G}_{p p}(\mathbf{r} ; \mathbf{n}(\mathbf{x}) ; \mathbf{l}(\mathbf{y}))= & \frac{G}{\pi} \frac{1}{r^{2}}\left[\mathbf{S Y M}(\mathbf{r} \otimes \mathbf{n}) \frac{\mathbf{r} \cdot \mathbf{l}}{r^{2}}+\mathbf{S Y M}(\mathbf{r} \otimes \mathbf{l}) \frac{\mathbf{r} \cdot \mathbf{n}}{r^{2}}\right] \\
& +\frac{G v}{\pi(1-v)} \frac{1}{r^{2}}\left\{\frac{\mathbf{r} \otimes \mathbf{r}}{r^{2}}\left[(\mathbf{l} \cdot \mathbf{n})-\frac{4}{v} \frac{(\mathbf{r} \cdot \mathbf{n})(\mathbf{r} \cdot \mathbf{l})}{r^{2}}\right]\right. \\
& \left.+\left[\frac{(\mathbf{r} \cdot \mathbf{n})(\mathbf{r} \cdot \mathbf{l})}{r^{2}}+\frac{(1-2 v)}{2 v}(\mathbf{l} \cdot \mathbf{n})\right] \mathbf{I}+\mathbf{S Y M}(\mathbf{l} \otimes \mathbf{n})\right\}
\end{aligned}
$$

For the 2D plane stress case, one needs to substitute $v$ with $v^{*}=v / 1+v$.

\section{APPENDIX B: BASIC LEBESGUE INTEGRALS}

The following identities, that can be proved by induction, when $r_{2} \neq 0$, are the keynote of the inner integration. Here, $\mathrm{M}_{2}[k]$ stands for the remainder of the (integer) division $k \div 2$.

$$
\begin{aligned}
\left.\int_{x_{1}-l_{j}}^{x_{1}+l_{j}} r_{1}^{k} \log \left(r^{2}\right) \mathrm{d} r_{1}\right|_{r_{2}=x_{2}}= & {\left[\log \left(r_{1}^{2}+x_{2}^{2}\right)\left(\frac{r_{1}^{k+1}}{k+1}+\mathrm{M}_{2}[k](-1)^{(k-1) / 2} x_{2}^{k+1}\right)\right.} \\
& +\arctan \left(\frac{r_{1}}{x_{2}}\right)\left(\left(1-\mathrm{M}_{2}[k]\right) \frac{2}{k-1}(-1)^{k / 2} x_{2}^{k+1}\right)
\end{aligned}
$$




$$
\begin{aligned}
& \left.+r_{1} \sum_{n=0}^{k} \mathrm{M}_{2}[k+1-n] \frac{2}{(k+1)(n+1)}(-1)^{((k-n) / 2+1)} r_{1}^{n} x_{2}^{k-n}\right]_{r_{1}=x_{1}-l_{j}}^{r_{1}=x_{1}+l_{j}} \\
& \left.\int_{x_{1}-l_{j}}^{x_{1}+l_{j}} \frac{r_{1}^{k}}{r^{2}} \mathrm{~d} r_{1}\right|_{r_{2}=x_{2}}=\left[\log \left(r_{1}^{2}+x_{2}^{2}\right)\left(\mathrm{M}_{2}[k](-1)^{(k-1) / 2} x_{2}^{k-1}\right)\right. \\
& +\arctan \left(\frac{r_{1}}{x_{2}}\right)\left(\left(1-\mathrm{M}_{2}[k]\right)(-1)^{k / 2} x_{2}^{k-1}\right) \\
& \left.+\sum_{n=1}^{k} \frac{\mathrm{M}_{2}[k-n]}{n}(-1)^{((k-n+1) / 2+1)} r_{1}^{n} x_{2}^{k-n-1}\right]_{r_{1}=x_{1}-l_{j}}^{r_{1}=x_{1}+l_{j}} \\
& \left.\int_{x_{1}-l_{j}}^{x_{1}+l_{j}} \frac{r_{1}^{k}}{r^{4}} \mathrm{~d} r_{1}\right|_{r_{2}=x_{2}}=\left[\frac{1}{2} \log \left(r_{1}^{2}+x_{2}^{2}\right)\left(-\frac{k-1}{2} \mathrm{M}_{2}[k](-1)^{(k-1) / 2} x_{2}^{k-3}\right)\right. \\
& +\frac{1}{2} \arctan \left(\frac{r_{1}}{x_{2}}\right)\left((k-1)\left(\mathrm{M}_{2}[k]-1\right)(-1)^{k / 2} x_{2}^{k-3}\right) \\
& +\frac{1}{2} \frac{1}{r^{2}} x_{2}^{k-2}\left(-\mathrm{M}_{2}[k](-1)^{(k-1) / 2} x_{2}+\left(1-\mathrm{M}_{2}[k]\right)(-1)^{k / 2} r_{1}\right) \\
& \left.+\sum_{n=1}^{k-2} \mathrm{M}_{2}[k-n] \frac{k-n-1}{2 n}(-1)^{(k-n+1) / 2} r_{1}^{n} x_{2}^{k-n-3}\right]_{r_{1}=x_{1}-l_{j}}^{r_{1}=x_{1}+l_{j}} \\
& \left.\int_{x_{1}-l_{j}}^{x_{1}+l_{j}} \frac{r_{1}^{k}}{r^{6}} \mathrm{~d} r_{1}\right|_{r_{2}=x_{2}}=\left[\frac{1}{4} \log \left(r_{1}^{2}+x_{2}^{2}\right)\left(\frac{k-3}{2} \frac{k-1}{2} \mathrm{M}_{2}[k](-1)^{(k-1) / 2} x_{2}^{k-5}\right)\right. \\
& +\frac{1}{4} \arctan \left(\frac{r_{1}}{x_{2}}\right)\left(\frac{k-3}{2}(k-1)\left(1-\mathrm{M}_{2}[k]\right)(-1)^{k / 2} x_{2}^{k-5}\right) \\
& +\frac{1}{4} \frac{1}{r^{4}} x_{2}^{k-2}\left(-\mathrm{M}_{2}[k](-1)^{(k-1) / 2} x_{2}+\left(1-\mathrm{M}_{2}[k]\right)(-1)^{k / 2} r_{1}\right) \\
& +\frac{1}{4} \frac{1}{r^{2}} x_{2}^{k-4}\left(\mathrm{M}_{2}[k](-1)^{(k-1) / 2}(k-1) x_{2}-\left(1-\mathrm{M}_{2}[k]\right)(-1)^{k / 2} \frac{2 k-3}{2} r_{1}\right) \\
& \left.+\frac{1}{8} \sum_{n=1}^{k-4} \mathrm{M}_{2}[k-n] \frac{k-n-1}{n}(k-n-3)(-1)^{(k-n-1) / 2} r_{1}^{n} x_{2}^{k-n-5}\right]_{r_{1}=x_{1}-l_{j}}^{r_{1}=x_{1}+l_{j}}
\end{aligned}
$$




\section{APPENDIX C}

In this appendix, tables $\mathbf{L}_{p p}, \mathbf{A}_{p p}, \mathbf{S}_{p p}, \mathbf{H}_{p p}, \mathbf{P}_{p p}, \mathbf{L}_{u u}, \mathbf{A}_{u u}, \mathbf{P}_{u u}, \mathbf{L}_{u p}, \mathbf{A}_{u p}, \mathbf{S}_{u p}, \mathbf{P}_{u p}, \mathbf{L}_{p u}, \mathbf{A}_{p u}$, $\mathbf{S}_{p u}$ and $\mathbf{P}_{p u}$, for quadratic shape functions are collected.

\section{C.1. Weak kernel}

$$
\begin{aligned}
& \mathbf{L}_{u u}[1,1]:=\left\{\frac{r_{1}(-3+4 v)}{2}, \frac{r_{1}^{2}(-3+4 v)+r_{2}^{2}(-5+4 v)}{4}, \frac{r_{1}^{3}(-3+4 v)}{6}\right\} \\
& \mathbf{L}_{u u}[1,2]:=\left\{\frac{r_{2}}{2}, 0, \frac{-r_{2}^{3}}{2}\right\} \quad \mathbf{L}_{u u}[2,1]:=\left\{\frac{r_{2}}{2}, 0, \frac{-r_{2}^{3}}{2}\right\} \\
& \mathbf{L}_{u u}[2,2]:=\left\{\frac{r_{1}(-3+4 v)}{2}, \frac{r_{1}^{2}(-3+4 v)+r_{2}^{2}(-1+4 v)}{4}, \frac{r_{1}^{3}(-3+4 v)}{6}\right\} \\
& \mathbf{A}_{u u}[1,1]:=\left\{4 r_{2}(-1+v), 0, \frac{2 r_{2}^{3}(3-2 v)}{3}\right\} \\
& \mathbf{A}_{u u}[1,2]:=\left\{0,-r_{2}^{2}, 0\right\} \quad \mathbf{A}_{u u}[2,1]:=\left\{0,-r_{2}^{2}, 0\right\} \\
& \mathbf{A}_{u u}[2,2]:=\left\{2 r_{2}(-1+2 v), 0, \frac{-4 r_{2}^{3} v}{3}\right\} \\
& \mathbf{P}_{u u}[1,1]:=\left\{-4 r_{1}(-1+v), \frac{r_{1}^{2}(5-4 v)}{4}, \frac{-2 r_{1}\left(r_{1}^{2}-3 r_{2}^{2}\right)(-3+2 v)}{9}\right\} \\
& \mathbf{P}_{u u}[1,2]:=\mathbf{P}_{u u}[2,1]:=\left\{0, r_{1} r_{2}, \frac{r_{1}^{2} r_{2}}{2}\right\} \\
& \mathbf{P}_{u u}[2,2]:=\left\{r_{1}(3-4 v), \frac{r_{1}^{2}(3-4 v)}{4}, \frac{r_{1}^{3}(3-4 v)+12 r_{1} r_{2}^{2} v}{9}\right\}
\end{aligned}
$$

C.2. Strongly singular kernels: $\mathbf{G}_{u p}$

$$
\begin{array}{ll}
\mathbf{L}_{u p}[1,1]:=\left\{0, r_{2}\left(\frac{3}{2}-v\right), 0\right\} & \mathbf{L}_{u p}[1,2]:=\left\{-\frac{1}{2}+v, 0, \frac{r_{2}^{2}(3-2 v)}{2}\right\} \\
\mathbf{L}_{u p}[2,1]:=\left\{\frac{1}{2}-v, 0, \frac{r_{2}^{2}(1+2 v)}{2}\right\} & \mathbf{L}_{u p}[2,2]:=\left\{0, \frac{r_{2}(1-2 v)}{2}, 0\right\} \\
\mathbf{A}_{u p}[1,1]:=\left\{2-2 v, 0,2 r_{2}^{2}(-2+v)\right\} & \mathbf{A}_{u p}[1,2]:=\left\{0,-2 r_{2}(-1+v), 0\right\} \\
\mathbf{A}_{u p}[2,1]:=\left\{0,2 r_{2} v, 0\right\} & \mathbf{A}_{u p}[2,2]:=\left\{2-2 v, 0,2 r_{2}^{2} v\right\} \\
\mathbf{S}_{u p}[1,1]:=\left\{-\left(r_{1} r_{2}\right), r_{2}^{3}, r_{1} r_{2}^{3}\right\} & \mathbf{S}_{u p}[1,2]:=\mathbf{S}_{u p}[2,1]=\left\{-r_{2}^{2},-\left(r_{1} r_{2}^{2}\right), r_{2}^{4}\right\}
\end{array}
$$




$$
\begin{array}{ll}
\mathbf{S}_{u p}[2,2]:=\left\{r_{1} r_{2},-r_{2}^{3},-\left(r_{1} r_{2}^{3}\right)\right\} & \\
\mathbf{P}_{u p}[1,1]:=\left\{0,0, r_{1} r_{2}(3-2 v)\right\} & \mathbf{P}_{u p}[1,2]:=\left\{0, r_{1}(-1+2 v), \frac{r_{1}^{2}(-1+2 v)}{2}\right\} \\
\mathbf{P}_{u p}[2,1]:=\left\{0, r_{1}-2 r_{1} v, \frac{r_{1}^{2}(1-2 v)}{2}\right\} & \mathbf{P}_{u p}[2,2]:=\left\{0,0, r_{1} r_{2}(1-2 v)\right\}
\end{array}
$$

\section{C.3. Strongly singular kernels: $\mathbf{G}_{p u}$}

$$
\begin{aligned}
& \mathbf{L}_{p u}[1,1]:=\left\{n_{1}\left(-\frac{3}{2}+v\right), \frac{n_{2} r_{2}(-3+2 v)}{2}, \frac{n_{1} r_{2}^{2}(5-2 v)}{2}\right\} \\
& \mathbf{L}_{p u}[1,2]:=\left\{n_{2}\left(-\frac{1}{2}+v\right), \frac{-\left(n_{1} r_{2}(1+2 v)\right)}{2}, \frac{-\left(n_{2} r_{2}^{2}(1+2 v)\right)}{2}\right\} \\
& \mathbf{L}_{p u}[2,1]:=\left\{\frac{n_{2}(1-2 v)}{2}, \frac{n_{1} r_{2}(-3+2 v)}{2}, \frac{n_{2} r_{2}^{2}(-3+2 v)}{2}\right\} \\
& \mathbf{L}_{p u}[2,2]:=\left\{n_{1}\left(-\frac{1}{2}+v\right), \frac{n_{2} r_{2}(-1+2 v)}{2}, \frac{-\left(n_{1} r_{2}^{2}(1+2 v)\right)}{2}\right\} \\
& \mathbf{A}_{p u}[1,1]:=\left\{2 n_{2}(-1+v),-2 n_{1} r_{2}(-2+v),-2 n_{2} r_{2}^{2}(-2+v)\right\} \\
& \mathbf{A}_{p u}[1,2]:=\left\{-2 n_{1} v,-2 n_{2} r_{2} v, 2 n_{1} r_{2}^{2}(1+v)\right\} \\
& \mathbf{A}_{p u}[2,1]:=\left\{2 n_{1}(-1+v), 2 n_{2} r_{2}(-1+v),-2 n_{1} r_{2}^{2}(-2+v)\right\} \\
& \mathbf{A}_{p u}[2,2]:=\left\{2 n_{2}(-1+v),-2 n_{1} r_{2} v,-2 n_{2} r_{2}^{2} v\right\} \\
& \mathbf{S}_{p u}[1,1]:=\left\{r_{2}\left(n_{2} r_{1}-n_{1} r_{2}\right),-r_{2}^{2}\left(n_{1} r_{1}+n_{2} r_{2}\right),-r_{2}^{3}\left(n_{2} r_{1}-n_{1} r_{2}\right)\right\} \\
& \mathbf{S}_{p u}[1,2]:=\left\{r_{2}\left(n_{1} r_{1}+n_{2} r_{2}\right), r_{2}^{2}\left(n_{2} r_{1}-n_{1} r_{2}\right),-r_{2}^{3}\left(n_{1} r_{1}+n_{2} r_{2}\right)\right\} \\
& \mathbf{S}_{p u}[2,1]:=\left\{r_{2}\left(n_{1} r_{1}+n_{2} r_{2}\right), r_{2}^{2}\left(n_{2} r_{1}-n_{1} r_{2}\right),-r_{2}^{3}\left(n_{1} r_{1}+n_{2} r_{2}\right)\right\} \\
& \mathbf{S}_{p u}[2,2]:=\left\{-r_{2}\left(n_{2} r_{1}-n_{1} r_{2}\right), r_{2}^{2}\left(n_{1} r_{1}+n_{2} r_{2}\right), r_{2}^{3}\left(n_{2} r_{1}-n_{1} r_{2}\right)\right\} \\
& \mathbf{P}_{p u}[1,1]:=\left\{0, n_{1} r_{1}(-3+2 v), \frac{r_{1}\left(n_{1} r_{1}+2 n_{2} r_{2}\right)(-3+2 v)}{2}\right\} \\
& \mathbf{P}_{p u}[1,2]:=\left\{0, n_{2} r_{1}(-1+2 v), \frac{r_{1}\left(n_{2} r_{1}(-1+2 v)-2 n_{1} r_{2}(1+2 v)\right)}{2}\right\} \\
& \mathbf{P}_{p u}[2,1]:=\left\{0, n_{2} r_{1}(1-2 v), \frac{r_{1}\left(2 n_{1} r_{2}(-3+2 v)+n_{2}\left(r_{1}-2 r_{1} v\right)\right)}{2}\right\} \\
& \mathbf{P}_{p u}[2,2]:=\left\{0, n_{1} r_{1}(-1+2 v), \frac{r_{1}\left(n_{1} r_{1}+2 n_{2} r_{2}\right)(-1+2 v)}{2}\right\}
\end{aligned}
$$




\section{C.4. Hypersingular kernel}

$$
\begin{aligned}
& \mathbf{L}_{p p}[1,1]:=\left\{0, n_{2},-6 n_{1} r_{2}\right\} \quad \mathbf{L}_{p p}[2,2]:=\left\{0, n_{2}, 2 n_{1} r_{2}\right\} \\
& \mathbf{L}_{p p}[1,2]:=\mathbf{L}_{p p}[2,1]=\left\{0, n_{1}, 2 n_{2} r_{2}\right\} \\
& \mathbf{A}_{p p}[1,1]:=\left\{0,-4 n_{1},-8 n_{2} r_{2}\right\} \quad \mathbf{A}_{p p}[2,2]:=\mathbf{0} \\
& \mathbf{A}_{p p}[1,2]:=\mathbf{A}_{p p}[2,1]=\left\{0,0,-8 n_{1} r_{2}\right\} \\
& \mathbf{S}_{p p}[1,1]:=\left\{-2 n_{2} r_{1}+6 n_{1} r_{2}, 8 r_{2}\left(n_{1} r_{1}+n_{2} r_{2}\right),-2 r_{2}^{2}\left(-5 n_{2} r_{1}+7 n_{1} r_{2}\right)\right\} \\
& \mathbf{S}_{p p}[1,2]:=\mathbf{S}_{p p}[2,1]=\left\{-2\left(n_{1} r_{1}+n_{2} r_{2}\right),-4 r_{2}\left(n_{2} r_{1}-2 n_{1} r_{2}\right), 10 r_{2}^{2}\left(n_{1} r_{1}+n_{2} r_{2}\right)\right\} \\
& \mathbf{S}_{p p}[2,2]:=\left\{-2\left(n_{2} r_{1}+n_{1} r_{2}\right),-4 r_{2}\left(n_{1} r_{1}+n_{2} r_{2}\right),-2 r_{2}^{2}\left(3 n_{2} r_{1}-5 n_{1} r_{2}\right)\right\} \\
& \mathbf{H}_{p p}[1,1]:=\left\{4 r_{2}^{2}\left(n_{2} r_{1}-n_{1} r_{2}\right),-4 r_{2}^{3}\left(n_{1} r_{1}+n_{2} r_{2}\right),-4 r_{2}^{4}\left(n_{2} r_{1}-n_{1} r_{2}\right)\right\} \\
& \mathbf{H}_{p p}[1,2]:=\mathbf{H}_{p p}[2,1]=\left\{4 r_{2}^{2}\left(n_{1} r_{1}+n_{2} r_{2}\right), 4 r_{2}^{3}\left(n_{2} r_{1}-n_{1} r_{2}\right),-4 r_{2}^{4}\left(n_{1} r_{1}+n_{2} r_{2}\right)\right\} \\
& \mathbf{H}_{p p}[2,2]:=\left\{-4 r_{2}^{2}\left(n_{2} r_{1}-n_{1} r_{2}\right), 4 r_{2}^{3}\left(n_{1} r_{1}+n_{2} r_{2}\right), 4 r_{2}^{4}\left(n_{2} r_{1}-n_{1} r_{2}\right)\right\} \\
& \mathbf{P}_{p p}[1,1]:=\mathbf{P}_{p p}[2,2]:=\left\{0,0,2 n_{2} r_{1}\right\} \\
& \mathbf{P}_{p p}[1,2]:=\mathbf{P}_{p p}[2,1]=\left\{0,0,2 n_{1} r_{1}\right\}
\end{aligned}
$$

\section{REFERENCES}

1. Hackbusch W. Integral Equations. Birkhaeuser Verlag: Basel, 1995.

2. Brebbia CA, Telles JCF, Wrobel LC. Boundary Element Techniques. Springer: Berlin, 1984.

3. Aliabadi MH. Boundary element formulations in fracture mechanics. Applied Mechanical Review 1997; 50: $83-96$.

4. Maier G, Frangi A. Symmetric boundary element method for 'discrete' crack modelling of fracture processes. Computer Assisted Mechanical and Engineering Science 1998; 5:201-226.

5. Maier G, Novati G, Cen Z. Symmetric boundary element method for quasi-brittle fracture and frictional contact problems. Computational Mechanics 1993; 13:74-89.

6. Gray LJ, Paulino GH. Symmetric Galerkin boundary integral formulation for interface and multizone problems. International Journal for Numerical Methods in Engineering 1997; 40:3085-3101.

7. Layton JB, Ganguly S, Balakrishna C, Kane JH. A symmetric Galerkin multizone boundary element formulation. International Journal for Numerical Methods in Engineering 1997; 40:2913-2931.

8. Salvadori A. Quasi brittle fracture mechanics by cohesive crack models and symmetric Galerkin boundary element method. PhD Thesis, Politecnico di Milano, Milano, 1999.

9. Abousleiman Y, Cheng AHD. Boundary element solution for steady and unsteady Stokes flow. Computer Methods in Applied Mechanics and Engineering 1994; 117:1-13.

10. Rizzo FJ. An integral equation approach to boundary value problems of classical elastostatics. Quarterly Applied Mathematics 1967; 40:83-95.

11. Sirtori S, Maier G, Novati G, Miccoli S. A Galerkin symmetric boundary-element method in elasticity, formulation and implementation. International Journal for Numerical Methods in Engineering 1992; 35: $255-282$.

12. Huber O, Lang A, Kuhn G. Evaluation of the stress tensor in 3D elastostatics by direct solving of hypersingular integrals. Computational Mechanics 1993; 12:39-50.

13. Mantič V. On computing boundary limiting values of boundary integrals with strongly singular and hypersingular kernels in 3D BEM for elastostatics. Engineering Analysis with Boundary Elements 1994; 13:115-134.

14. Hong KH, Chen JT. Derivations of integral equations of elasticity. Journal of Engineering Mechanics ASCE 1988; 114(6):1028-1044. 
15. Diligenti M, Monegato G. Finite-part integrals, their occurence and computation. Rendiconti del Circolo Matematico di Palermo 1993; 33(II):39-61.

16. Guiggiani M. Hypersingular boundary integral equations have an additional free term. Computational Mechanics $1995 ; 16: 245-248$

17. Hadamard J. Lectures on Cauchy's Problem in Linear Partial Differential Equations. Yale Univ. Press: New Haven, Conn, 1923.

18. Krishnasamy G, Rizzo FJ, Rudolphi TJ. Hypersingular Boundary Integral Equations, Their Occurrence, Interpretation, Regularization and Computation. In Developments in Boundary Element Methods, vol. 7, Banerjee PK, Kobayashi S (eds). Elsevier Applied Science Publishers: Amsterdam, 1991.

19. Toh K, Mukherjee S. Hypersingular and finite part integrals in the boundary element method. International Journal of Solid Structures 1994; 31:2299-2312.

20. Mantič V. A new formula for the C-matrix in the Somigliana identity. Journal of Elasticity 1993; 33:191-201.

21. Hartmann F. The Somigliana identity on a piecewise smooth surface. Journal of Elasticity 1981; 11(4): $403-423$

22. Nedelec JC, Planchard J. Une methode variationelle d'elements finis pour la resolution numerique d'un problem exterieur dans $\mathbf{R}^{3}$. RAIRO Analyse Numerique 1973; 7:105-129.

23. Hsiao GC, Wendland WL. A finite element method for some integral equations of the first kind. Journal of Mathematical Analysis and Applications 1977; 58:449-481.

24. Sirtori S. General stress analysis by means of integral equation and boundary elements. Meccanica 1979; 14: $210-218$

25. Hartmann F, Katz C, Protopsaltis B. Boundary elements and symmetry. Ingenieur-Archiv 1985; 55:440-449.

26. Wendland WL. Variational methods for BEM. In Boundary Integral Methods (Theory and Applications), Morino L, Piva R (eds). Springer: Berlin, 1990.

27. Polizzotto C, Zito M. A variational approach to boundary element methods. In Boundary Elements Methods in Applied Mechanics, Tanaka M, Cruse T (eds). Pergamon Press: Oxford, 1989; 13-24.

28. Carini A, Salvadori A. Implementation of a symmetric Galerkin boundary element method in quasi-brittle fracture mechanics. Proceeding of IUTAM/IACM/IABEM 1999 Symposium. Kluwer Academic Press: Dordrecht, MA, 1999.

29. Polizzotto C. An energy approach to the boundary element method. Part I, Elastic solids. Computer Methods in Applied Mechanics and Engineering 1988; 69:167-184.

30. Bonnet M, Maier G, Polizzotto C. Symmetric Galerkin boundary element method. Applied Mechanical Review 1998; 51:669-704.

31. Kane JH, Balakrishna C. Symmetric Galerkin boundary formulations employing curved elements. International Journal for Numerical Methods in Engineering 1993; 36:2157-2187.

32. Rudolphi TJ. The use of simple solutions in the regularization of hypersingular boundary integral equations. Mathematical Computational Model 1991; 15:269-278.

33. Bonnet M. A regularized Galerkin symmetric BIE formulation for mixed 3D elastic boundary values problems. Boundary Elements Abstracts and Newsletters 1993; 4:109-113.

34. Sladek V, Sladek J. Transient elastodynamic three dimensional problems in cracked bodies. Applied Mathematical Model 1984; 8:2-10.

35. Frangi A, Novati G. Symmetric BE method in two-dimensional elasticity, evaluation of double integrals for curved elements. Computational Mechanics 1996; 19:58-68.

36. Frangi A, Novati G. Regularized symmetric Galerkin BIE formulations in the Laplace transform domain for 2D problems. Computational Mechanics 1998; 22:50-60.

37. Kutt HR. The numerical evaluation of principal value integrals by finite-part integration. Numerical Mathematics 1975; 24:205-210.

38. Monegato G. The numerical evaluation of hypersingular integrals. Journal of Computational and Applied Mathematics 1994; 50:9-31

39. Aimi A. New numerical integration schemes for solution of (hyper) singular integral equations with Galerkin BEM. PhD Thesis. Universita' degli Studi di Milano, Milano, 1997.

40. Balakrishna C, Gray LJ, Kane JH. Efficient analytic integration of symmetric Galerkin boundary integrals over curved elements, thermal conduction formulation. Computational Mathematics and Applied Mechanics in Engineering 1994; 111:335-355.

41. Aimi A, Carini A, Diligenti M, Monegato G. Numerical integration schemes for evaluation of (hyper)singular integrals in 2D BEM. Computational Mechanics 1998; 22:1-11.

42. Aimi A, Diligenti M, Monegato G. New numerical integration schemes for applications of Galerkin Bem to 2D problems. International Journal for Numerical Methods in Engineering 1997; 40:1977-1999.

43. Aimi A, Diligenti M, Monegato G. Numerical integration schemes for the BEM solution of hypersingular integral equations. International Journal for Numerical Methods in Engineering 1999; 45:1807-1830.

44. Elliott D, Paget DF. Gauss type quadrature rules for Cauchy principal value integrals. Mathematics of Computation 1979; 33(145):301-309. 
45. Guiggiani M, Krishnasamy G, Rudolphi TJ, Rizzo FJ. A general algorithm for the numerical solution of hypersingular boundary integral equations. Journal of Applied Mechanics 1992; 59:604-614.

46. Holzer S. How to deal with hypersingular integrals in the symmetric BEM. Communications and Applied Numerical Methods 1993; 9:219-232.

47. Hui CY, Shia D. Evaluations of hypersingular integrals using Gaussian quadrature. International Journal for Numerical Methods in Engineering 1999; 44:205-214.

48. Guiggiani M. Hypersingular formulation for boundary stress evaluation. Engineering Analysis with Boundary Elements 1994; 13:169-179.

49. Gray LJ, Martha LF, Ingraffea AR. Hypersingular integrals in boundary element fracture analysis. International Journal for Numerical Methods in Engineering 1990; 29:1135-1158.

50. Gray LJ, Soucie CS. A Hermite interpolation algorithm for hypersingular boundary integrals. International Journal for Numerical Methods in Engineering 1993; 36:2357-2367.

51. Carini A, Diligenti M, Maranesi P, Zanella M. Analytical integrations for two-dimensional elastic analysis by the symmetric Galerkin boundary element method. Computational Mechanics 1999; 23(4):308-323.

52. Mori M. Quadrature formulas obtained by variable transformation and the DE-Rule. Journal of Computational and Applied Mathematics 1985; 12:119-130.

53. Monegato G, Scuderi L. Numerical integration of functions with boundary singularities. Journal of Computational and Applied Mathematics 1999; 112:201-214.

54. Schwab C, Wendland WL. Kernel properties and representations of boundary integral operators. Mathematische Nachrichten 1998; 156:187-218.

55. Zemanian AH. Distribution Theory and Transform Analysis. Dover: New York, 1987.

56. Corradi Dell'Acqua L. Meccanica delle Strutture. McGraw-Hill: Milano, 1992 (in Italian).

57. Banerjee PK, Butterfield R. Boundary Element Method in Engineering Science. McGraw-Hill: New York, 1981.

58. Press WH, Flannery BP, Teukolsky SA, Vetterling WT. Numerical Recipes. The Art of Scientific Computing. Cambridge University Press: Cambridge, 1986.

59. Carini A, De Donato O. Fundamental solutions for linear viscoelastic continua. International Journal for Solids Structures 1992; 29:2989-3009. 